\title{
A survey of Floer homology for manifolds with contact type boun- dary or symplectic homology
}

\author{
Alexandru Oancea
}

\begin{abstract}
The purpose of this paper is to give a survey of the various versions of Floer homology for manifolds with contact type boundary that have so far appeared in the literature. Under the name of "Symplectic homology" or "Floer homology for manifolds with boundary" they bear in fact common features and we shall try to underline the principles that unite them. Once this will be accomplished we shall proceed to describe the peculiarity of each of the constructions and the specific applications that unfold out of it: classification of ellipsoids and polydiscs in $\mathbb{C}^{n}$, stability of the action spectrum for contact type boundaries of symplectic manifolds, existence of closed characteristics on contact type hypersurfaces. The computation of the Floer cohomology for balls in $\mathbb{C}^{n}$ is carried out by explicitly perturbing the nondegenerate Morse-Bott spheres of closed characteristics.
\end{abstract}

\section{Constructions of Floer homological invariants}

\subsection{Morse homology}

Floer homology can be seen as a vast generalization of the Thom-Smale-Witten (TSW) complex to infinite dimension. We briefly recall below the construction of the latter [F3, Sch]. The resulting homology theory is called Morse (co)homology as a tribute to Marston Morse's pioneering use of critical points of smooth maps in the study of the topology of manifolds.

Consider a Morse function $f: L \longrightarrow \mathbb{R}$ defined on a closed manifold $L$ and a Riemannian metric $g$ which is generic with respect to a certain transversality property. One defines two complexes, one homological and the other one

2000 Mathematics Subject Classification: 53D40, 37J45.

Work supported by ENS Lyon, Ecole Polytechnique (France) and ETH Zürich (Switzerland). 
cohomological, by

$$
\begin{aligned}
& C_{q}^{\text {Morse }}\left(L,-\nabla^{g} f\right)=\bigoplus_{\begin{array}{c}
\nabla^{g} f(x)=0 \\
\operatorname{ind}_{f}(x)=q
\end{array}} \mathbb{Z}\langle x\rangle, \\
& \partial^{\text {Morse }}: C_{q}^{\mathrm{Morse}}\left(L,-\nabla^{g} f\right) \longrightarrow C_{q-1}^{\mathrm{Morse}}\left(L,-\nabla^{g} f\right) \text {, } \\
& \partial^{\text {Morse }}\langle x\rangle=\sum_{\operatorname{ind}_{f}(y)=q-1} \#\left(\mathcal{M}\left(x, y ;-\nabla^{g} f\right) / \mathbb{R}\right)\langle y\rangle . \\
& C_{\text {Morse }}^{q}\left(L,-\nabla^{g} f\right)=\bigoplus_{\begin{array}{c}
\nabla^{g} f(x)=0 \\
\operatorname{ind}_{f}(x)=q
\end{array}} \mathbb{Z}\langle x\rangle \\
& \delta_{\text {Morse }}: C_{\text {Morse }}^{q}\left(L,-\nabla^{g} f\right) \longrightarrow C_{\text {Morse }}^{q+1}\left(L,-\nabla^{g} f\right) \text {, } \\
& \delta_{\text {Morse }}\langle x\rangle=\sum_{\operatorname{ind}_{f}(y)=q+1} \#\left(\mathcal{M}\left(y, x ;-\nabla^{g} f\right) / \mathbb{R}\right)\langle y\rangle .
\end{aligned}
$$

The notation $\mathcal{M}\left(y, x ;-\nabla^{g} f\right)$ stands for the space of parameterized negative gradient trajectories running from $y$ to $x$, on which the additive group $\mathbb{R}$ naturally acts by reparameterization. The transversality condition mentioned above concerns the transverse intersection of any pair of stable and unstable manifolds of $-\nabla^{g} f$. It ensures in particular that $\mathcal{M}\left(y, x ;-\nabla^{g} f\right)$ is a smooth manifold of dimension $\operatorname{ind}_{f}(y)-\operatorname{ind}_{f}(x)$, where $\operatorname{ind}_{f}(x)$ is the Morse index of the nondegenerate critical point $x$ for the function $f$. When the difference of the indices is equal to 1 , a careful description of the relative compactness of sequences of trajectories proves that the quotient $\mathcal{M}\left(y, x ;-\nabla^{g} f\right) / \mathbb{R}$ is finite. Any choice of orientations for the unstable manifolds allows one to orient the trajectory spaces and algebraically count the elements of $\mathcal{M}\left(y, x ;-\nabla^{g} f\right) / \mathbb{R}$ when $\operatorname{ind}_{f}(y)-\operatorname{ind}_{f}(x)$ is equal to 1 . This gives a precise meaning for the expressions defining $\partial^{\text {Morse }}$ and $\delta_{\text {Morse }}$. The crucial identities $\left(\partial^{\text {Morse }}\right)^{2}=0$ and $\left(\delta_{\text {Morse }}\right)^{2}=0$ are a consequence of a glueing theorem which constitutes, together with the analysis of the convergence of sequences of trajectories, a description of the compactification of $\mathcal{M}\left(y, x ;-\nabla^{g} f\right) / \mathbb{R}$ by "broken trajectories". Finally, the homology of the above complexes is seen to compute the singular homology and, respectively, cohomology $H_{*}\left(C_{*}^{\text {Morse }}\left(L,-\nabla^{g} f\right)\right) \simeq H_{*}(L ; \mathbb{Z})$, $H^{*}\left(C_{\text {Morse }}^{*}\left(L,-\nabla^{g} f\right)\right) \simeq H^{*}(L ; \mathbb{Z})$. The most intuitive way to see this is to use the fact $[\mathrm{L}]$ that the unstable manifolds give rise to a $\mathrm{CW}$-decomposition of $L$ and use cellular (co)homology as a bridge between Morse and singular (co)homology [Po, O]. 
We should mention at this point two important extensions of the preceding setup. Firstly, the negative gradient vector field can be replaced by a vector field $X$ which is negative gradient-like with respect to a Morse function $f$ i.e. $X \cdot f<0$ outside the critical points of $f$, which satisfies the same transversality condition and which is equal to $-\nabla^{g} f$ near the critical points for some given metric $g$. Secondly, when the manifold $L$ is no longer compact, the same construction can be carried out if $f$ satisfies the Palais-Smale condition: every sequence $\left(x_{\nu}\right)$ such that $f\left(x_{\nu}\right)$ is bounded and $\nabla^{g} f\left(x_{\nu}\right) \longrightarrow 0$ contains a convergent subsequence. The Palais-Smale condition can of course be formulated for a (negative) gradient-like vector field. For any two regular values $a<b$ of $f$ the complexes constructed on the critical points belonging to $f^{-1}[a, b]$ eventually compute the relative singular homology/cohomology $H_{*}\left(C_{*}^{\text {Morse }}\left(L,-\nabla^{g} f ; a, b\right)\right) \simeq H_{*}\left(f^{b}, f^{a} ; \mathbb{Z}\right), H^{*}\left(C_{\text {Morse }}^{*}\left(L,-\nabla^{g} f ; a, b\right)\right) \simeq$ $H^{*}\left(f^{b}, f^{a} ; \mathbb{Z}\right)$. For any real number $c$ we have denoted $f^{c}=\{f \leq c\}$.

\subsection{Floer homology for closed manifolds}

Let us now describe the main lines along which Hamiltonian Floer homology of a closed manifold is constructed. This section recalls the construction presented in F. Laudenbach's paper "Symplectic geometry and Floer homology" published in this same issue of the journal. A comprehensive reference is also provided by D. Salamon's lecture notes [S2], while the full details under the symplectic asphericity condition $\left\langle\omega, \pi_{2}(M)\right\rangle=0$ that we require henceforth are disseminated in several papers of A. Floer [F1, F2]. This assumption can be eliminated with moderate effort in the monotone case ("bubbles") and with strenuous one ("virtual cycle technique") in the general case, but it is suitable for this survey paper to simplify things. We henceforth assume $\left\langle\omega, \pi_{2}(M)\right\rangle=0$, as well as $\left\langle c_{1}, \pi_{2}(M)\right\rangle=0$ in order for the Conley-Zehnder index of a periodic orbit to be well defined.

Let $H: \mathbb{S}^{1} \times M \longrightarrow \mathbb{R}$ be a time-dependent Hamiltonian and $X_{H}$ the Hamiltonian vector field defined by $\iota_{X_{H}} \omega=d H(t, \cdot)$. Let $J_{t} \in \operatorname{End}(T M), t \in \mathbb{S}^{1}$ be a loop of almost complex structures which are compatible with the symplectic form i.e. $g_{J_{t}}(\cdot, \cdot)=\omega\left(\cdot, J_{t} \cdot\right), t \in \mathbb{S}^{1}$ are symmetric positive definite bilinear forms. We then have $J_{t} X_{H}(t, \cdot)=\nabla^{g_{J_{t}}} H(t, \cdot)$.

From a formal point of view, the construction of Floer homology closely follows that of Morse homology with the following analogies:

Manifold $L \quad \longleftrightarrow$ Space of contractible loops in $M$, denoted $\Lambda$

$$
\begin{array}{r}
\text { Metric } g \longleftrightarrow L^{2} \text { metric on } \Lambda:\langle\eta, \xi\rangle=\int_{\mathbb{S}^{1}} g_{J_{t}}(\eta(t), \xi(t)) d t, \\
\eta, \xi \in T_{\gamma} \Lambda=C^{\infty}\left(\mathbb{S}^{1}, \gamma^{*} T M\right)
\end{array}
$$


Function $f \longleftrightarrow$ Action functional $A_{H}: \Lambda \longrightarrow \mathbb{R}$

$$
\begin{gathered}
\gamma \longmapsto-\int_{D^{2}} \bar{\gamma}^{*} \omega-\int_{\mathbb{S}^{1}} H(t, \gamma(t)) d t \\
d f(x) \longleftrightarrow d A_{H}(\gamma) \cdot \eta=-\int_{\mathbb{S}^{1}} \omega\left(\eta(t), \dot{\gamma}(t)-X_{H}(\gamma(t))\right) d t
\end{gathered}
$$

Critical point of $f \longleftrightarrow 1$ - periodic orbit: $\dot{\gamma}(t)=X_{H}(t, \gamma(t))$

$$
\nabla^{g} f \longleftrightarrow \nabla^{J} A_{H}(\gamma)=J_{t} \dot{\gamma}-J_{t} X_{H}
$$

Negative gradient trajectory $\longleftrightarrow$ Map $u: \mathbb{R} \longrightarrow \Lambda$ or $u: \mathbb{R} \times \mathbb{S}^{1} \longrightarrow M$ satisfying

$$
\frac{\partial u}{\partial s}+J_{t}(u(s, t)) \frac{\partial u}{\partial t}-\nabla^{g_{J_{t}}} H(t, u(s, t))=0
$$

and having bounded energy

$$
E(u)=\frac{1}{2} \int_{\mathbb{S}^{1}} \int_{\mathbb{R}}\left(\left|\frac{\partial u}{\partial s}\right|^{2}+\left|\frac{\partial u}{\partial t}-X_{H}(t, u)\right|^{2}\right) d s d t<\infty .
$$

Let us make some comments before going on with the dictionary. For a contractible loop $\gamma: \mathbb{S}^{1} \longrightarrow M$ we have denoted $\bar{\gamma}: D^{2} \longrightarrow M$ a map verifying $\left.\bar{\gamma}\right|_{\mathbb{S}^{1}}=\gamma$. The symplectic asphericity implies that the action functional is well defined i.e. the quantity $\int_{D^{2}} \bar{\gamma}^{*} \omega$ does not depend on the choice of the extension $\bar{\gamma}$.

From a formal point of view, the analogues of the negative gradient trajectories of the finite dimensional case are maps $u: \mathbb{R} \times \mathbb{S}^{1} \longrightarrow M$ only verifying equation (1.4). For finite-dimensional closed manifolds this automatically implies the convergence at $\pm \infty$ to critical points of $f$. On the contrary, when the dimension is infinite one has to impose a supplementary condition in order to ensure the convergence at $\pm \infty$ to 1-periodic orbits. This condition is precisely given by the finiteness of the energy (1.5). The latter is equivalent to the convergence at $\pm \infty$ of a cylinder verifying (1.4), under the assumption that the 1-periodic orbits of $H$ are nondegenerate. For a 1-periodic orbit $x(t)=x(t+1)$, this means that

$$
\operatorname{det}\left(\operatorname{Id}-d \varphi_{H}^{1}(x(0))\right) \neq 0
$$

where $\varphi_{H}^{t}$ is the flow of $X_{H}$.

The minima of the energy are precisely the solutions of Floer's equation (1.4). For any two orbits $x, y$ of period 1 let us denote by $\mathcal{U}(y, x)$ the space of maps $u: \mathbb{R} \times \mathbb{S}^{1} \longrightarrow M$ that verify $\lim _{s \rightarrow-\infty} u(s, t)=y(t), \lim _{s \rightarrow+\infty} u(s, t)=x(t)$ with uniform convergence in $t$. Any element in $u \in \mathcal{U}(y, x)$ satisfies 


$$
E(u)=\frac{1}{2} \int_{\mathbb{S}^{1}} \int_{\mathbb{R}}\left|\frac{\partial u}{\partial s}+J_{t}(u) \frac{\partial u}{\partial t}-\nabla H(t, u)\right|^{2} d s d t+A_{H}(y)-A_{H}(x) .
$$

This is a consequence of the identities $-\frac{d}{d s} A_{H}(u(s, \cdot))=\int_{\mathbb{S}^{1}} \omega\left(u_{s}, u_{t}-X_{H}\right) d t$ and $\left|u_{s}+J u_{t}-J X_{H}\right|^{2}=\left|u_{s}\right|^{2}+\left|u_{t}-X_{H}\right|^{2}-2 \int_{\mathbb{S}_{1}} \omega\left(u_{s}, u_{t}-X_{H}\right) d t$. The minimum $A_{H}(y)-A_{H}(x)$ of the energy on $\mathcal{U}(y, x)$ is attained precisely by the solutions of equation (1.4) and we denote $\mathcal{M}(y, x)$ the set of these trajectories. An element of $\mathcal{M}(y, x)$ will be called a Floer trajectory.

A similar phenomenon occurs in the finite dimensional case: the negative gradient trajectories are the minima of the energy $E_{f, g}(\gamma)=\frac{1}{2} \int_{\mathbb{R}}|\dot{\gamma}|^{2}+\left|\nabla^{g} f(\gamma)\right|^{2} d s$, defined on the space of maps $\gamma: \mathbb{R} \longrightarrow L$ that converge at $\pm \infty$ to some given critical points of $f$. The transversality condition that was mentioned in $\$ 1.1$ can be rephrased in terms of a surjectivity property for the linearization of the equation of gradient trajectories $[\mathrm{S} 1]$ :

$$
D_{\gamma} \xi=\nabla_{s} \xi+\nabla_{\xi} \nabla f(\gamma), \quad \xi \in \gamma^{*} T L .
$$

A similar transversality condition has to be verified in the infinite dimensional case in order for the spaces $\mathcal{M}(y, x)$ to inherit the structure of a finite dimensional manifold. The linearization

$$
D_{u} \xi=\nabla_{s} \xi+J(u) \nabla_{t} \xi+\nabla_{\xi} J(u) u_{t}-\nabla_{\xi} \nabla H(t, u), \quad \xi \in u^{*} T M
$$

of the equation (1.4) has to be a Fredholm map whose differential is surjective at solutions of (1.4). The Fredholm character requires the use of suitable functional spaces for the elliptic analysis and holds if the 1-periodic orbits of $H$ are nondegenerate. The surjectivity is satisfied for a generic choice of $H$ and $J$ [FHS]. Our dictionary goes now as follows:

Nondegenerate critical points $\longleftrightarrow 1$ - periodic nondegenerate orbits

Morse index $\operatorname{ind}_{\text {Morse }}(x) \longleftrightarrow$ Conley-Zehnder index $i_{C Z}(\gamma)$ of a of a critical point $x \quad$ periodic orbit $\gamma$, taken with negative sign

$$
\begin{array}{r}
\operatorname{dim} \mathcal{M}(y, x)=\operatorname{ind}_{\text {Morse }}(y)-\operatorname{ind}_{\text {Morse }}(x) \longleftrightarrow \operatorname{dim} \mathcal{M}(y, x)=i_{C Z}(x)-i_{C Z}(y) \\
=-i_{C Z}(y)-\left(-i_{C Z}(x)\right)
\end{array}
$$

Let us remark that, unlike in the finite dimensional case, the Hessian of $A_{H}$ at a critical point admits an infinite number of negative as well as positive eigenvalues. Its "Morse" index is therefore not well defined. This can be easily seen on the following example: consider $H \equiv 0$ on $\mathbb{C}$ and the action of a loop $\gamma=$ $\sum_{k \in \mathbb{Z}} z_{k} e^{i k t}$ is $\int x d y=\pi \sum_{k \in \mathbb{Z}} k\left|z_{k}\right|^{2}$. The positive and negative eigenspaces of 
this quadratic form are obviously infinite dimensional. In fact, this shows again that the classical Morse theory is not adapted to the present context: it would come to glueing infinite dimensional discs along infinite dimensional spheres, which are contractible. The homotopical invariants of the corresponding spaces would vanish at any step of the construction.

The Conley-Zehnder index is an integer that is associated to a path of symplectic matrices having the identity as origin and whose end does not contain the eigenvalue 1 in its spectrum. To any periodic orbit $\gamma$ one can associate a Conley-Zehnder index by trivializing $T M$ over a filling disc $\bar{\gamma}$ and by considering the path of symplectic matrices induced by the linearization of the Hamiltonian flow along $\gamma$. The assumption $\left\langle c_{1}, \pi_{2}(M)\right\rangle=0$ ensures that two such trivializations are homotopically equivalent along $\gamma$ and the integer $i_{C Z}(\gamma)$ will thus be independent of the trivialization. We explain at the end of this section why the suitable analogue of the Morse index is the Conley-Zehnder index considered with negative sign, rather than simply the Conley-Zehnder index.

If the transversality assumptions are verified, the implicit function theorem ensures that the dimension of $\mathcal{M}(y, x)$ at $u$ is equal to the Fredholm index of $D_{u}$. The identification of the latter with the difference between the ConleyZehnder indices of the ends is a consequence of a characterization in terms of the spectral flow of a certain family of first order differential operators that is associated to equation (1.4) [RS1, RS2, S2].

The only ingredient still lacking in order to formally define a (co)homological differential complex in analogy with the TSW complex is a recipe to associate a sign to a Floer trajectory running between periodic orbits whose ConleyZehnder indices have a difference equal to 1 . We shall not pursue this matter here and will just claim that there is one such recipe [FH2, S1]. One can alternatively work with $\mathbb{Z} / 2 \mathbb{Z}$ coefficients in order to avoid all sign problems.

Definition. The homological Floer complex $F C_{*}(M ; H, J)$ and the cohomological one $F C^{*}(M ; H, J)$ are defined respectively by the analogues of equations $(1.1,1.2,1.3)$ and $\left(1.1^{\prime}, 1.2^{\prime}, 1.3^{\prime}\right)$ through the above dictionary. The grading is given by the opposite of the Conley-Zehnder index.

The definition depends on the transversality results that we have mentioned above. In the case of a Hamiltonian having nondegenerate periodic orbits, the almost complex structures for which these hold form a set $\mathcal{J}_{\text {reg }}(H)$ which is of the second category in the sense of Baire in the space of $\omega$-compatible almost complex structures. Conversely, for any fixed family $J=\left(J_{t}\right)$, the transversality results are valid for a second Baire category set of Hamiltonians $\mathcal{H}_{\text {reg }}(J) \subset C^{\infty}\left(\mathbb{S}^{1} \times M, \mathbb{R}\right)$. In finite dimension, this amounts to prescribe the metric and to choose a generic Morse function: from the point of view of genericity, the metric and the function play symmetric roles.

In order to fix ideas, all the remarks that are to follow will concern cohomology groups. The distinction homology - cohomology will gain importance only for manifolds with boundary. The definition will in that case contain as 
a supplementary ingredient an algebraic limit process which, according to the formalism being homological or cohomological, will be direct or inverse.

The fundamental property of Floer cohomology

$$
F H^{*}(M ; H, J)=H^{*}\left(F C^{*}(M ; H, J)\right)
$$

is its independence with respect to the Hamiltonian and with respect to the almost complex structure. For any two pairs $\left(H^{0}, J^{0}\right),\left(H^{1}, J^{1}\right)$ which satisfy the above regularity conditions there is a homotopy of regular pairs $\left(H^{t}, J^{t}\right)$, $t \in[0,1]$ that links them together. Any such homotopy induces an isomorphism $F H^{*}\left(M ; H^{0}, J^{0}\right) \stackrel{\sim}{\longrightarrow} F H^{*}\left(M ; H^{1}, J^{1}\right)$ which, moreover, does not depend on the chosen regular homotopy. The consequence is that one can identify Floer (co)homology with a classical topological invariant, namely singular (co)homology. Let us recall the relevant argument. Consider a Hamiltonian function that is time independent and Morse. Any critical point $x$ of $H$ is a (constant) 1-periodic orbit and, in view of the convention $X_{H}=-J \nabla H$, we infer $i_{C Z}(x)=n-\operatorname{ind}_{\text {Morse }}(x,-H), n=\frac{1}{2} \operatorname{dim} M$ or, written in a different way, $\operatorname{ind}_{\text {Morse }}(x,-H)=n+\left(-i_{C Z}(x)\right)$. When $H$ is small enough in the $C^{2}$ norm one can show that there are no 1-periodic orbits other than the critical points of $H$ and, moreover, the Floer trajectories (solving $u_{s}+J u_{t}=\nabla H$ ) that run between points whose index difference is equal to 1 are in fact time independent [F2]. This means that the Floer complex coincides with the TSW complex corresponding to $\nabla H$. If the grading on $F C^{*}$ is given by minus the Conley-Zehnder index, one gets the isomorphism

$$
F H^{*}(M, \omega) \simeq H^{n+*}(M ; \mathbb{Z}), \quad n=\frac{1}{2} \operatorname{dim} M .
$$

We see in particular the interest of grading by $-i_{C Z}$ rather than $i_{C Z}$. In the latter case we would have obtained the isomorphism $F H^{*} \simeq H^{n+*}$ through the extra use of Poincaré duality $H_{n-*}(M) \simeq H^{n+*}(M)$.

\subsection{Floer homology for manifolds with contact type boun- dary or Symplectic homology}

The second big setting in which Hamiltonian Floer homology groups can be defined is that of compact symplectic manifolds with contact type boundary. The main references on this topic are the papers by K. Cieliebak, A. Floer, H. Hofer, K. Wysocki [FH1, CFH, CFHW] and C. Viterbo [V2]. I have used D. Hermann's thesis [He1] with great profit due to the very clear exposition style. The paper of P. Biran, L. Polterovich and D. Salamon [BPS] contains results concerning the existence of periodic orbits representing nontrivial free homotopy classes. The Weinstein conjecture and related problems are discussed in the book by H. Hofer and E. Zehnder [HZ].

The initial motivation for the construction of Floer homology groups was the existence problem for closed orbits of Hamiltonian systems. Roughly speaking, 
this problem has two distinct aspects: existence of closed orbits with a given period and existence of closed orbits on a given energy level. Historically, these two directions correspond to two conjectures of V.I. Arnold [A] (1965) and A. Weinstein [We] (1979). In one of its variants, the first claims that a lower bound for the number of closed orbits with fixed period on a closed symplectic manifold $M$ is provided by the rank of the (rational) cohomology $H^{*}(M ; \mathbb{Q})$. Under the assumptions $\left\langle\omega, \pi_{2}(M)\right\rangle=0,\left\langle c_{1}(M), \pi_{2}(M)\right\rangle=0$ of the previous section, this follows from the very construction of the Floer homology groups. Since 1996 there are proofs that work in full generality, with no extra assumptions on the underlying manifold. The second conjecture claims the existence of at least one closed orbit on a regular compact energy level $\Sigma$ which is of contact type. Floer homology for manifolds with boundary is a tool that is particularly adapted to the study of this question $(\S 4.3)$, but we shall present two other fascinating applications in $\S 4.1$ and $\S 4.2$.

Definition 1.1. A (compact) hypersurface $\Sigma$ of a symplectic manifold $(M, \omega)$ is said to be of contact type if there is a vector field $X$ defined in a neighbourhood of $\Sigma$, transverse to $\Sigma$ and verifying $L_{X} \omega=\omega$. The vector field $X$ is called the Liouville field. The 1-form $\lambda=\iota_{X} \omega$ is called the Liouville form. If the Liouville field is globally defined on the whole of $M$ we say that $\Sigma$ is of restricted contact type.

The boundary of a compact symplectic manifold $M$ is said to be of (restricted) contact type if the above conditions are satisfied and the Liouville field is outward pointing.

The contact type condition is a symplectic analogue for convexity in the linear symplectic space $\mathbb{R}^{2 n}$ : any compact convex hypersurface is of (restricted) contact type, as the radial vector field $X(x)=\frac{1}{2} x, x \in \mathbb{R}^{2 n}$ satisfies the above conditions (assuming 0 is in the bounded component of $\mathbb{R}^{2 n} \backslash \Sigma$ ). The conjecture has been formulated precisely in view of preliminary existence results on convex or star-shaped energy levels. A first proof for a contact type $\Sigma \subset \mathbb{R}^{2 n}$ was given by C. Viterbo [V1] and lots of other ambient spaces have been subsequently exlored.

The contact type condition is related to holomorphic pseudo-convexity, as remarked by Y. Eliashberg, M. Gromov [EG] and D. McDuff [McD]. This is precisely the reason why we impose that the Liouville field be outward pointing. One should remark that this is automatically true if the boundary is of restricted contact type as the Liouville field (exponentially) expands volumes. We give more details on pseudo-convexity in the sequel.

Floer homology groups of a manifold $M$ with contact type boundary will be defined with the help of Hamiltonians that admit the boundary $\partial M$ as a regular level. The invariants that we thus obtain will take into account not only the 1periodic orbits in the interior of the manifold, but also the closed orbits having arbitrary period on the boundary. As a consequence, they are well adapted to the study of Weinstein's problem. Let us stress from the very beginning that, unlike Floer homology of closed manifolds which is finally proved to be equal 
to the singular homology, Floer homology of manifolds with boundary has no similar topological correspondent. It is all the more important to exhibit in the latter case qualitative properties that are determined by additional geometric properties of the manifold.

Here is how one retrieves closed orbits of arbitrary period on a contact type level with the help of 1-periodic orbits in a neighbourhood of $\Sigma$. Note that the restriction of $\omega$ to $T \Sigma$ has a one dimensional kernel on which $\lambda$ does not vanish. If $H$ is an autonomous Hamiltonian admitting $\Sigma$ as a regular level then $\left.X_{H} \in \operatorname{ker} \omega\right|_{T \Sigma}$ and $\lambda\left(X_{H}\right) \neq 0$.

Definition 1.2. The Reeb vector field (or characteristic field) $X_{\text {Reeb }}$ of $\Sigma$ is defined by the following two properties: $\left.X_{\text {Reeb }} \in \operatorname{ker} \omega\right|_{T \Sigma}$ and $\lambda\left(X_{\text {Reeb }}\right)=1$. An orbit of $X_{\text {Reeb }}$ is called a characteristic of $\Sigma$.

One should note that the area $\int_{\gamma} \lambda$ of a closed characteristic is equal to its period. Moreover, the orbits of $X_{H}$ that are located on $\Sigma$ are in one-to-one correspondence with the latter's characteristics. It is important to understand that the Hamiltonian dynamics on a regular level does not depend on the Hamiltonian but on the level itself: it is more of a geometric nature rather than purely dynamic or analytic.

Let us denote by $\varphi_{X}^{t}$ the flow of the Liouville field. A whole neighbourhood $\mathcal{V}$ of $\Sigma$ is foliated by the hypersurfaces $\varphi_{X}^{t}(\Sigma)_{-\delta<t<\delta}$ with $\delta>0$ small enough. In view of $\varphi_{X}^{t}{ }^{*} \omega=e^{t} \omega$, the characteristics on these hypersurfaces are in one to one correspondence with those of $\Sigma$. It is now comfortable to make a coordinate change via the symplectic diffeomorphism

$$
\begin{gathered}
\Psi: \Sigma \times[1-\delta, 1+\delta] \stackrel{\sim}{\longrightarrow} \mathcal{V}, \quad \delta>0 \text { small }, \\
\Psi(p, S)=\varphi_{X}^{\ln (S)}(p)
\end{gathered}
$$

verifying

$$
\Psi^{*} \lambda=S \cdot \lambda \mid
$$

where $\lambda \mid$ is the restriction of $\lambda=\iota_{X} \omega$ to $T \Sigma$. The autonomous Hamiltonian

$$
\begin{aligned}
& H: \Sigma \times[1-\delta, 1+\delta] \longrightarrow \mathbb{R} \\
& H(p, S)=h(S), \quad h:[1-\delta, 1+\delta] \longrightarrow \mathbb{R}
\end{aligned}
$$

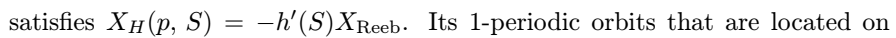
the level $S$ correspond to characteristics of period $h^{\prime}(S)$ located on $\Sigma$ (with the opposite orientation). By studying the 1-periodic orbits of such Hamiltonians one will have in fact studied characteristics on $\Sigma$ : the more important the variation of $h$ in the small interval $[1-\delta, 1+\delta]$ is, the more characteristics one "sees".

Any reasonable Floer homology invariant for a manifold with boundary should take into account the topology of the manifold and all closed characteristics on 
the boundary. We thus retrieve the common underlying principle of the constructions in [FH1, CFH, CFHW, V2]: the cohomology

$$
F H^{*}(M)=\lim _{(H, J)} F H^{*}(M ; H, J)
$$

will be defined as a limit following an admissible family of Hamiltonians, steeper and steeper near the boundary. As we have already warned the reader, this limit is of direct or inverse type respectively, according to the choice of a homological or cohomological formalism. One supplementary refinement will consist in using a truncation by the values of the action, a crucial ingredient for the applications in $\S 4.1$ and $\S 4.2$. Before reaching them, let us describe the various points of view already present in the literature.

Symplectic homology of a bounded open set $U \subset\left(\mathbb{C}^{n}, \omega_{0}\right)$, cf. [FH1]

This definition was introduced by A. Floer and H. Hofer [FH1]. In order to give a presentation in tune with the subsequent constructions we shall present below the cohomological setup, while the original point of view is homological. The two main features of this theory are the following:

a. it is "extrinsic" in the sense that Hamiltonians are defined on the whole ambient space $\mathbb{C}^{n}$

b. the definition is valid for arbitrary open sets, without any regularity or contact type hypothesis on the boundary.

The class $\mathcal{H}(U)$ of admissible Hamiltonians $H: \mathbb{S}^{1} \times \mathbb{C}^{n} \longrightarrow \mathbb{R}$ is defined by the following properties:

1. $H_{\left.\right|_{\mathbb{S}^{1} \times \bar{U}}}<0$;

2. there is a positive definite matrix $A$ such that

$$
\frac{\left|H^{\prime}(t, u)-A u\right|}{|u|} \longrightarrow 0, \quad|u| \rightarrow \infty
$$

uniformly in $t \in \mathbb{S}^{1}$;

3. the differential system

$$
-i \dot{x}=A x, \quad x(0)=x(1)
$$

admits only the trivial solution $x \equiv 0$;

4. there is a constant $c>0$ such that

$$
\begin{gathered}
\left\|H^{\prime \prime}(t, u)\right\| \leq c, \quad \forall t \in \mathbb{S}^{1}, u \in \mathbb{C}^{n}, \\
\left|\frac{\partial H^{\prime}}{\partial t}(t, u)\right| \leq c(1+|u|), \quad \forall t \in \mathbb{S}^{1}, u \in \mathbb{C}^{n} .
\end{gathered}
$$


Condition (1) "pins down" the Hamiltonian along $U$ and allows it to (steeply) increase only in the neighbourhood of the boundary, according to the philosophy that we have detailed above. Condition (2) prescribes the asymptotic behaviour of $X_{H}$ and, combined with (3), ensures not only that all its 1-periodic orbits are contained in a compact set, but also that the Floer trajectories that link them together do not escape to infinity. This is a crucial point in proving Floer compactness for open manifolds and we shall give more details on it in the context of the two constructions to follow. Condition (4) is of a technical nature and plays some role in the proof of the $C^{0}$ estimates. One should note that condition (2) is to be interpreted as a kind of quadratic asymptoticity for $H$.

We denote by $\mathcal{H}_{\text {reg }}(U)$ the class of admissible Hamiltonians having nondegenerate 1-periodic orbits: it is of second Baire category in $\mathcal{H}(U)$. We denote by $\mathcal{J}$ the class of almost complex structures on $\mathbb{C}^{n}$ that are compatible with $\omega_{0}$ and that are equal to the standard complex structure $i$ outside a compact set. The transversality in Floer's equation is verified for a dense set $\mathcal{H}_{\text {reg }}(U) \subset \mathcal{H}_{\text {reg }}(U) \times \mathcal{J}$ and the truncated Floer cohomology groups are defined for a regular pair as follows:

$$
\begin{gathered}
F C_{] a,+\infty[}^{k}(H, J)=\bigoplus_{\substack{-i C Z(x)=k \\
A_{H}(x)>a}} \mathbb{Z}\langle x\rangle, \quad a \in \mathbb{R} \cup\{-\infty\}, \\
F H_{] a,+\infty[}^{*}(H, J)=H^{*}\left(F C_{] a,+\infty[}^{*}(H, J)\right), \\
F C_{] a, b]}^{*}(H, J)=F C_{] a,+\infty[}^{*}(H, J) / F C_{] b,+\infty[}^{*}(H, J),-\infty \leq a<b<+\infty, \\
F H_{] a, b]}^{*}(H, J)=H^{*}\left(F C_{] a, b]}^{*}(H, J)\right) .
\end{gathered}
$$

The inverse limit (1.7) is considered with respect to the following partial order relation on $\mathcal{H J}_{\text {reg }}(U)$, which induces an inverse system on the cohomology groups:

$$
(H, J) \prec(K, \widetilde{J}) \quad \text { iff } \quad H(t, u) \leq K(t, u) .
$$

The cohomological inverse system is determined in the following manner. For two ordered pairs $(H, J) \prec(K, \widetilde{J})$ we consider a homotopy $(H(s, t, u)$, $J(s, t, u))$ such that:

- There is an $s_{0}>0$ with $(H(s, t, u), J(s, t, u)) \equiv(H(t, u), J(t, u)), s \leq-s_{0}$ $(K(t, u), \widetilde{J}(t, u)), s \geq s_{0}$;

- The homotopy is an increasing function with respect to $s$ i.e. $\frac{\partial H}{\partial s}(s, t, u) \geq 0$;

- The homotopy satisfies certain additional assumptions concerning the behaviour at infinity and the regularity. The most important of them is the following compatibility with the quadratic asymptoticity: 
There is a smooth path $A(s) \in \operatorname{End}_{\mathbb{R}}\left(\mathbb{C}^{n}\right)$ of positive definite matrices such that $A(s)=A\left(-s_{0}\right)$ for $s \leq-s_{0}, A(s)=A\left(s_{0}\right)$ for $s \geq s_{0}$ and

$$
\frac{\left|H^{\prime}(s, t, u)-A(s) u\right|}{|u|} \longrightarrow 0, \quad|u| \rightarrow \infty .
$$

Moreover, we require that if the Hamiltonian system $-i \dot{x}=A(\hat{s}) x$ has a nontrivial 1-periodic solution for some $\hat{s} \in \mathbb{R}$, then $\left.\frac{d}{d s} A(s)\right|_{s=\hat{s}}$ is positive definite.

The asymptotic conditions on the homotopy ensure that the solutions of the parameterized Floer equation

$$
\begin{gathered}
u_{s}+J(s, t, u) u_{t}-\nabla H(s, t, u)=0, \\
u(s, t) \longrightarrow x^{ \pm}, s \longrightarrow \pm \infty,
\end{gathered}
$$

which play now the role of the trajectories verifying $(1.4-1.5)$, do also stay in a compact set. Here $x^{-}$and $x^{+}$are 1-periodic orbits of $H$ and $K$ respectively. The dimension of the moduli space $\mathcal{M}\left(x^{-}, x^{+}\right)$of solutions of $(1.8-1.9)$ is $-i_{C Z}\left(x^{-}\right)-\left(-i_{C Z}\left(x^{+}\right)\right)$but, to the difference of equation $(1.4-1.5)$, the group $\mathbb{R}$ is no longer acting by translation. This implies that, generically, the moduli space is no longer empty if its formal dimension is zero and the morphism of complexes defined according to $\left(1.3^{\prime}\right)$ will respect the grading:

$$
\begin{gathered}
\sigma: F C_{] a, \infty]}^{*}(K, \widetilde{J}) \longrightarrow F C_{] a, \infty]}^{*}(H, J), \\
\sigma\left\langle x^{+}\right\rangle=\sum_{i_{C Z}\left(x^{-}\right)=i_{C Z}\left(x^{+}\right)} \# \mathcal{M}\left(x^{-}, x^{+}\right)\left\langle x^{-}\right\rangle .
\end{gathered}
$$

The map $\sigma$ is well defined because the action is decreasing along solutions of the parameterized equation (1.8). This is due to the fact that the homotopies that we allow are increasing:

$$
\begin{aligned}
\frac{d}{d s} A_{H(s)}(u(s, \cdot)) & =-\int_{\mathbb{S}^{1}} \omega\left(u_{s}, u_{t}-X_{H(s)}(u(s, t))\right) d t-\int_{\mathbb{S}^{1}} \frac{\partial H}{\partial s}(s, t, u(s, t)) d t \\
& =-\int_{\mathbb{S}^{1}} \omega\left(u_{s}, J(s, t, u(s, t)) u_{s}\right) d t-\int_{\mathbb{S}^{1}} \frac{\partial H}{\partial s}(s, t, u(s, t)) d t \\
& =-\left\|u_{s}\right\|_{g_{J(s)}}^{2}-\int_{\mathbb{S}^{1}} \frac{\partial H}{\partial s}(s, t, u(s, t)) d t \leq 0 .
\end{aligned}
$$

The morphism $\sigma$ commutes with the differential and descends to a morphism in cohomology that is called "monotonicity map"

$$
F H_{] a, b]}^{*}(K, \widetilde{J}) \stackrel{\sigma_{(H, J)}^{(K, \widetilde{J})}}{\longrightarrow} F H_{] a, b]}^{*}(H, J)
$$


As in the compact case, two admissible homotopies induce the same morphism in homology and we moreover have

$$
\sigma_{(H, J)}^{(K, \widetilde{J})} \circ \sigma_{(K, \widetilde{J})}^{\left(K^{\prime}, \widetilde{J}^{\prime}\right)}=\sigma_{(H, J)}^{\left(K^{\prime}, \widetilde{J}^{\prime}\right)}
$$

for $(H, J) \prec(K, \widetilde{J}) \prec\left(K^{\prime}, \widetilde{J}^{\prime}\right)$. Let us then define

$$
\begin{aligned}
& F H_{] a, b]}^{*}(U)=\lim _{(\overleftarrow{H, J})} F H_{] a, b]}^{*}(H, J), \quad-\infty \leq a<b<+\infty, \\
& F H_{] a,+\infty[}^{*}(U)=\lim _{(\overleftarrow{H, J})} F H_{] a,+\infty[}^{*}(H, J)
\end{aligned}
$$

and get in particular

$$
F H_{] a,+\infty[}^{*}(U)=\lim _{\overleftarrow{b}} F H_{] a, b]}^{*}(U), \quad b \rightarrow+\infty .
$$

The "truncation maps" $F H_{] a, b]}^{*}(U) \longrightarrow F H_{\left.] a, b^{\prime}\right]}^{*}(U), b \geq b^{\prime}$ are induced by the inclusions $F C_{] a, \infty[}^{*}(H, J) \hookrightarrow F C_{] a^{\prime}, \infty[}^{*}(H, J), a \geq a^{\prime}$ which give rise to the morphisms

$$
F H_{] a, b]}^{*}(H, J) \longrightarrow F H_{] a^{\prime}, b^{\prime}\right]}^{*}(H, J), \quad a \geq a^{\prime}, b \geq b^{\prime} .
$$

The latter are compatible with the monotonicity maps. For a fixed value of $a$ the groups $F H_{] a, b]}^{*}(H, J)$ form an inverse bi-directed system. In view of the fact that two inverse (or direct) limits in a bi-directed system commute one can therefore write

$F H_{] a,+\infty[}^{*}(U)=\lim _{\overleftarrow{b}} \lim _{(\overleftarrow{H, J})} F H_{] a, b]}^{*}(H, J)=\lim _{(\overleftarrow{H, J})} \lim _{b} F H_{] a, b]}^{*}(H, J), \quad b \rightarrow \infty$

We shall see below (cf. $\S 2.2$ ) that, for an open set $U$ with restricted contact type boundary, the groups $F H_{] a,+\infty}^{*}(U)$ do not depend on $a$ if the latter is strictly negative. This is due to the existence of a cofinal family whose 1-periodic orbits all have an action that is either positive, either negative and arbitrarily close to zero [He1, V2]. We define in the general case

$$
F H^{*}(U)=F H_{-\infty,+\infty[}^{*}(U) .
$$

We shall then have $F H^{*}(U)=F H_{] a,+\infty[}^{*}(U), a<0$ if $U$ has restricted contact type boundary.

As we have already pointed out, the present version of Floer homology asks for an important new ingredient compared to the case of closed manifolds, namely the existence of a priori $C^{0}$ bounds which ensure that, for fixed limiting orbits, all Floer trajectories for the parameterized equation stay in a compact set. If this were not the case then some pathological noncompactness might appear in the moduli space of Floer trajectories: the number of trajectories between 
two orbits with index difference equal to one might be infinite, or the square of the Floer differential might no longer be zero. We give more details about the proofs of these bounds for the two constructions to follow and in $\S 2.1$.

Let us mention that an analogous construction can be used to define Floer homology groups. The differential will be defined according to (1.3) (and this is the original setup of $[\mathrm{FH} 1])$. The sub-complexes that will be preserved by the differential are

$$
F C_{*}^{]-\infty, a]}(H, J)=\bigoplus_{A_{H}(x)<a} \mathbb{Z}\langle x\rangle, \quad a \leq \infty
$$

and the monotonicity and truncation morphisms will define a direct double system:

$F H_{*}^{] a,+\infty[}(U)=\lim _{\vec{b}} \lim _{(\overrightarrow{H, J})} F H_{*}^{] a, b]}(H, J)=\underset{(\overrightarrow{H, J})}{\lim _{\vec{b}}} \underset{\vec{b}}{\lim } F H_{] a, b]}^{*}(H, J), \quad b \rightarrow \infty$.

This distinction bears a specific importance: the direct limit is an exact functor, while the inverse limit is generally only left exact and becomes exact if the terms of the directed system are all finite dimensional vector spaces ([ES] Ch. VIII). This has nontrivial consequences in practice: one can prove for example a Künneth formula that is valid in Floer homology with arbitrary coefficients, while the analogous Künneth formula holds in Floer cohomology only with coefficients in a field (see $\S 4.3)$.

\section{Symplectic homology of relatively compact open sets in manifolds with contact type boundary, cf. [CFH] and [CFHW]}

We describe below a construction introduced by K. Cieliebak, A. Floer and H. Hofer in $[\mathrm{CFH}]$, as well as one of its variants appearing in [CFHW].

The admissible Hamiltonians for a relatively compact open set $U \subset M \backslash \partial M$ are defined in $[\mathrm{CFH}]$ by the following properties:

$-H_{\left.\right|_{S^{1} \times \bar{U}}}<0$

- $H \equiv m(H)=\max H>0$ in a neighbourhood of $\partial M$;

- all periodic orbits satisfying $\int_{0}^{1} H(t, x(t)) d t<m(H)$ are nondegenerate.

A neighbourhood of the boundary is trivialized by the Liouville flow $\varphi \circ \ln$ as $(\partial M \times[1-\delta, 1], d(S \lambda \mid)), \delta>0$. The admissible almost complex structures are defined to be those that can be written near the boundary as

$$
\left\{\begin{array}{l}
J_{(p, S)} \mid \xi=J_{0} \\
J_{(p, S)}\left(\frac{\partial}{\partial S}\right)=\frac{1}{C S} X_{\text {Reeb }}(p), \quad C>0 \\
J_{(p, S)}\left(X_{\text {Reeb }}(p)\right)=-C S \frac{\partial}{\partial S} .
\end{array}\right.
$$


with $J_{0}$ an almost complex structure compatible with the restriction of $\omega$ to the contact distribution $\xi$ on $\partial M$. These are precisely the almost complex structures that are invariant by homotheties $(p, S) \longmapsto(p, a S), a>0$. The homotopies are again chosen to be increasing in the $s$-variable and such that, for fixed $s$, the Hamiltonian $H(s, \cdot, \cdot)$ is of the type above.

The definitions of the Floer complex and of the (co)homology groups are perfectly similar to those that we have described for open sets in $\mathbb{C}^{n}$ and the existence of the $C^{0}$ bounds for the parameterized trajectories is again the crucial point. In the present situation it is replaced by the requirement that the parameterized Floer trajectories stay at bounded distance from the boundary. To the difference of the preceeding construction and thanks to the very special form of the Hamiltonians and of the homotopies, a geometric argument based on holomorphic convexity allows one to conclude easily.

Definition 1.3. Let $J$ an almost complex structure that is compatible with the symplectic form $\omega$. A hypersurface $\Sigma \subset M$ is said to be $J$-convex if it can be locally written as the regular level set of a plurisubharmonic function i.e. a function $\varphi: M \longrightarrow \mathbb{R}$ which satisfies $d d^{c} \varphi \leq 0$, where $d^{c}=J^{*} d$.

Example: if $M$ is a manifold with contact type boundary, all hypersurfaces $\partial M \times\left\{S_{0}\right\} \subset \partial M \times[1-\epsilon, 1]$ are $J$-convex with respect to any admissible almost complex structure. Indeed, $\varphi(p, S)=S$ is plurisubharmonic as $d d^{c} S=$ $d(-C S \lambda \mid)=-C \omega \leq 0$.

The interest of plurisubharmonic functions and pseudo-convex hypersurfaces lies in the following lemma.

Lemma 1.4. (H. Hopf, see also [McD]) Let $\Sigma \subset M$ be a J-convex hypersurface and $\varphi$ a (local) function of definition which is plurisubharmonic. No Jholomorphic curve $u:\left(D^{2}(0,1), i\right) \longrightarrow M$ can have an interior strict tangency point with $\Sigma$ i.e. $\varphi \circ u$ cannot have a strict local maximum.

Proof. Let $z=s+i t$ be the complex coordinate on $D^{2}(0,1)$ and $J_{0}=i$ be the standard complex structure on the disk. The crucial (easy) computation is $d d_{J_{0}}^{c}(\varphi \circ u)=-\Delta(\varphi \circ u) d s \wedge d t$. The $J$-holomorphicity of $u$ implies $d d_{J_{0}}^{c}(\varphi \circ$ $u) \stackrel{J_{0}}{=} d J_{0}^{*} u^{*} d \varphi=d u^{*} J^{*} d \varphi=u^{*} d d_{J}^{c} \varphi$. But $\varphi$ is plurisubharmonic and thus $\Delta(\varphi \circ u) \geq 0$ i.e. $\varphi \circ u$ is plurisubharmonic in the classical sense. Hence $\varphi \circ u$ satisfies the mean value inequality and there can be no strict maximum that is achieved in the interior of the disk.

One can refine this argument and prove that $\varphi \circ u$ can neither have an interior maximum, be it strict or not. This shows that all Floer trajectories corresponding to a fixed Hamiltonian stay at a bounded distance from the boundary. The argument requires some further refinement in order to deal with parameterized trajectories but its basic feature remains the use of the maximum principle [CFH]. 
In order to get an intuition of what the corresponding invariants really compute, let us consider the case where the open set $U$ has a contact type boundary as well. One can then construct a cofinal family of Hamiltonians with a special geometric meaning, whose form is sketched in Figure 1 (2). A neighbourhood of $\partial U$ is trivialized by the corresponding Liouville flow as $\partial U \times[1-\epsilon, 1+\epsilon]$ and the Hamiltonians are of the form $H=h\left(S^{\prime}\right), S^{\prime} \in[1-\epsilon, 1+\epsilon]$, with $h$ steeper and steeper (see $\S 1.3$ for a discussion of nondegeneracy). Characteristics on $\partial U$ are therefore seen twice, once on the convex part and once on the concave part of the function $h$.

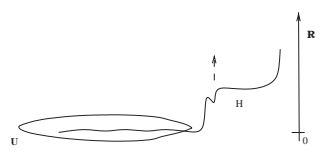

(1)

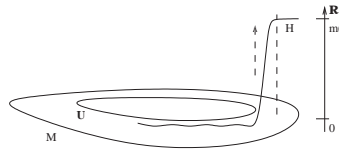

(2)

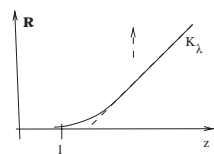

(3)

Figure 1: Admissible families: (1) - [FH1], (2) - [CFH], (3) - [V2]

Here is now the slight modification of the above definition which is used in $[\mathrm{CFHW}]$ for proving the stability of the action spectrum for a compact symplectic manifold with contact type boundary. We describe it in view of its use in $\S 4.2$, as well as in order to further discuss the relevance of the direction in which morphisms go in Floer (co)homology. One should note that this definition produces an invariant of the whole manifold and that there are no open sets involved anymore.

In this new setting, a periodic Hamiltonian $H: \mathbb{S}^{1} \times M \longrightarrow \mathbb{R}$ is admissible if it satisfies the following:

$-H \leq 0$

$-H \equiv 0$ in a neighbourhood of $\partial M$;

- all periodic orbits $x$ of $X_{H}$ satisfying $\int_{x} H<0$ are nondegenerate.

There is one important remark that has to be made concerning this setting: by definition of the admissible Hamiltonians, they have plenty of degenerate 1periodic orbits, namely constants in a neighbourhood of $\partial M$ with action equal to 0 . This has as consequence the fact that the truncated homology groups $F H_{a, b]}^{*}$ cannot possibly be well defined if $\left.\left.0 \in\right] a, b\right]$. On the other hand, by using admissible almost complex structures of the type described above they are well defined if $a<b<0$ or $0 \leq a<b$. The maximum principle applies in this context in a direct way to the parameterized Floer trajectories (which are holomorphic) near the boundary and Lemma 1.4 directly gives the fact that (parameterized) trajectories stay at a bounded distance from $\partial M$. 
As we now want to let the admissible Hamiltonians go to $-\infty$, the partial order will be

$$
H \prec K \quad \text { iff } \quad H(t, x) \geq K(t, y) .
$$

If we stick to the cohomological formalism, the inverse limit that we have previously encountered will therefore change to a direct limit. Note the difference with $[\mathrm{CFHW}]$ where a slight error concerning this point has found its way in the paper, with no consequences whatsoever.

\section{Floer homology of manifolds with contact type boundary}

This approach has been developed by C. Viterbo in [V2]. In comparison with the previous ones, it directly isolates the specific features of Floer (co)homology that are linked to the Weinstein conjecture by building a complex in which to every characteristic corresponds a single (transversally nondegenerate) generator. We define the symplectic completion of $M$ as

$$
\begin{gathered}
\widehat{M}=M \cup_{\partial M \times\{1\}} \partial M \times[1,+\infty[, \\
\widehat{\omega}=\left\{\begin{array}{cl}
\omega & \text { on } M \\
d(S \lambda \mid) & \text { on } \partial M \times[1,+\infty[.
\end{array}\right.
\end{gathered}
$$

The glueing is realized through the diffeomorphism (1.6) induced by the Liouville flow. Formally, the admissible Hamiltonians are of the type $K_{\mu}, \mu>0$ such that $K_{\mu} \equiv 0$ on $M, K_{\mu}(t, p, S)=k_{\mu}(S)$ on $\partial M \times[1,+\infty)$, with $k_{\mu}^{\prime}(S)=\mu$ for $S \geq 1^{+}$and $k_{\mu}$ convex. We have sketched a typical graph in Figure 1 (3). The homotopies are chosen to be increasing in the $s$ variable and convex. We also note at this point that it would be enough to choose Hamiltonians that are linear in the $S$ variable outside a compact set or linear at infinity.

The 1-periodic orbits of $K_{\mu}$ are the constants in the interior of $M$ and the closed characteristics on $\partial M$ having an action at most equal to $\mu$. As $K_{\mu}$ is time independent, any nonconstant orbit is at most transversally nondegenerate and one will have to think in practice to a perturbed Hamiltonian or family of Hamiltonians $\widetilde{K}_{\mu}$. Full details concerning the perturbations are provided in D. Hermann's thesis [He1] and the technique of perturbation is due to [FHW]: a nonconstant and transversally nondegenerate periodic orbit is to be seen as a Morse-Bott critical nondegenerate circle and a small time-dependent perturbation supported in a neighbourhood will "break" the circle into two nondegenerate periodic orbits, corresponding to the critical points of a Morse function on $\mathbb{S}^{1}$. More explanations on this point of view are given in section 3 , where we perform the same kind of perturbation for Morse-Bott nondegenerate spheres, not only circles. The perturbed Hamiltonians $\widetilde{K}_{\mu}$ satisfy

$-\widetilde{K}_{\mu} \leq \widetilde{K}_{\mu^{\prime}}$ if $\mu \leq \mu^{\prime}$;

- $\left(\widetilde{K}_{\mu}\right)_{\mu>0}$ is a cofinal family among the Hamiltonians $H: \mathbb{S}^{1} \times \widehat{M} \longrightarrow \mathbb{R}$ which verify $H<0$ on $M \backslash \partial M$. 
The admissible almost complex structures are chosen to be of the form (1.13) out of a compact set. The existence of $C^{0}$ bounds comes from the fact that, for generic values of $\mu$, there are no characteristics of period $\mu$ on $\partial M$. As a consequence, the 1-periodic orbits of $K_{\mu}$ are all located in a neighbourhood of $M$. The Floer trajectories that connect such 1-periodic orbits could leave the respective neighbourhood only by having an interior tangency with a certain $\partial M \times\left\{S_{0}\right\}$, a phenomenon which is forbidden by the Lemma below, based again on the maximum principle.

Lemma 1.5. [V2] Any solution $u:\left(D^{2}(0,1), i\right) \longrightarrow \partial M \times[1-\epsilon, \infty[$ of Floer's equation

$$
u_{s}+J u_{t}-\nabla H(s, t, u(s, t))=0
$$

with $J$ a standard almost complex structure, $H(s, t, p, S)=h(s, t, S)$ and $\frac{\partial^{2} h}{\partial s \partial S} \geq 0$ cannot have a strict interior tangency with some $\partial M \times\left\{S_{0}\right\}$.

Proof. In what follows we shall denote by $h^{\prime}, h^{\prime \prime}$ the derivatives of $h$ with respect to $S$. We put $f=S \circ u$ and we show that it cannot have an interior strict maximum point. One first computes

$$
\nabla H(s, t, u)=C f h^{\prime}(s, t, f) \frac{\partial}{\partial S},
$$

in view of $\left|\frac{\partial}{\partial S}\right|^{2}=\frac{1}{C S}$. We therefore have $u_{s}+J u_{t}-C f h^{\prime} \frac{\partial}{\partial S}=0$ which, by applying $d S$ and $J$, gives

$$
\partial_{s} f-C(S \circ u) \lambda\left(u_{t}\right)-C f h^{\prime}=0
$$

and

$$
\partial_{t} f+C(S \circ u) \lambda\left(u_{s}\right)=0 .
$$

We apply $\frac{\partial}{\partial s}$ to the first equation, $\frac{\partial}{\partial t}$ to the second and sum up:

$$
\begin{aligned}
0 & =\Delta f-C\left(\partial_{s}\left((S \circ u) \lambda_{u}\left(u_{t}\right)\right)-\partial_{t}\left((S \circ u) \lambda_{u}\left(u_{s}\right)\right)\right)-C \partial_{s}\left(f h^{\prime}\right) \\
& =\Delta f-C\left(u_{s}\left((S \lambda)\left(u_{t}\right)\right)-u_{t}\left((S \lambda)\left(u_{s}\right)\right)\right)-C \partial_{s}\left(f h^{\prime}\right) \\
& =\Delta f-C\left(d(S \lambda)\left(u_{s}, u_{t}\right)-(S \lambda)\left(\left[u_{s}, u_{t}\right]\right)\right)-C \partial_{s}\left(f h^{\prime}\right) \\
& =\Delta f-C\left(\left|u_{s}\right|^{2}-d(S \lambda)\left(u_{s}, h^{\prime} X_{\mathrm{Reeb}}\right)\right)-C \partial_{s}\left(f h^{\prime}\right) \\
& =\Delta f-C\left|u_{s}\right|^{2}+C d S\left(u_{s}\right) h^{\prime}-C \partial_{s} f h^{\prime}-C f \frac{\partial^{2} h}{\partial s \partial S}-C f h^{\prime \prime} \partial_{s} f \\
& =\Delta f-C\left|u_{s}\right|^{2}-C f \frac{\partial^{2} h}{\partial s \partial S}-C f h^{\prime \prime} \partial_{s} f .
\end{aligned}
$$

We have used $\left[u_{s}, u_{t}\right]=0$ and $u_{t}=J u_{s}-h^{\prime} X_{\text {Reeb }}$ in the fourth equality. We finally get

$$
\Delta f-C f h^{\prime \prime}(s, t, f) \partial_{s} f=C\left|u_{s}\right|^{2}+C f \frac{\partial^{2} h}{\partial s \partial S} \geq 0
$$


with the last inequality holding precisely due to the hypothesis $\frac{\partial^{2} h}{\partial s \partial S} \geq 0$. Thus $f$ satisfies the elliptic inequation of second order without zero order term

$$
\Delta f-C f h^{\prime \prime}(s, t, f) \partial_{s} f \geq 0,
$$

which obviously implies that $f$ cannot have an interior strict maximum.

We define as above the groups $F H_{\mid a,+\infty}^{*}(M),-\infty \leq a<\infty$. The new phenomenon is that they are independent of $a$ as soon as $a<0$. The explanation is very simple: the terms of the cofinal family that we consider all have 1 periodic orbits with action bigger than $-\delta$, with $\delta>0$ arbitrarily fixed. Indeed, the orbits are of two types: on the one hand the critical points of $H$ in the interior of $M$, with negative action close to zero, on the other hand the orbits corresponding to closed characteristics on $\partial M$, whose action is approximately equal to the (positive) area of the latter ones. One should bear in mind at this point that the action of a 1-periodic orbit appearing on a level $S$ is

$$
A=S h^{\prime}(S)-h(S) .
$$

We therefore have

$$
F H^{*}(M)=F H_{] a,+\infty[}^{*}(M), \quad a<0 .
$$

\section{Comments and further properties}

\section{$2.1 \quad C^{0}$ bounds}

As we have pointed out repeatedly, the crucial ingredient in the construction of a Floer (co)homology theory for manifolds with boundary is the existence of a priori $C^{0}$ bounds for Floer trajectories. The geometric pseudo-convexity argument of [V2] has to be modified in a nontrivial manner if one wishes to extend the class of admissible Hamiltonians and deformations by allowing a more general dependance on $s$ or $p$ in $H(s, t, p, S)$. This motivates the additional work in [FH1] and [CFH], on which we have not given full details. Nevertheless, some variant of the maximum principle enters in both contexts: [FH1] Prop. 8 and $[\mathrm{CFH}]$ p. 110. We mention that the ideas in [FH1], pp. 48-56 can be adapted to the setting of [V2] in order to establish the $C^{0}$ bounds for "asymptotically linear Hamiltonians" (see [O] $\S 1.2$ ).

\subsection{Relations between different symplectic homologies}

D. Hermann [He2] $\S 4.3$ proves that the homology groups defined in [FH1] for a bounded open set $U \subset \mathbb{C}^{n}$ coincide with the ones defined in [V2] if $U$ has 
restricted contact type boundary. Moreover, this holds for any fixed range of the action. The proof goes by exhibiting a cofinal sequence of Hamiltonians (Figure 2) that are admissible in the sense of [FH1] and whose periodic orbits fall in two classes: on one side orbits having positive or close to zero action (of type I and II), on the other side orbits whose action tends uniformly to $-\infty$ and which therefore do not count in the Floer complex (of type III, IV and $\mathbf{V}$ ). This is possible with a suitable choice of the parameters $A, B, \lambda$ and $\mu$. The cofinal sequence is constructed so that the orbits of the first kind are also orbits of a Hamiltonian that is admissible in the sense of [V2] and the isomorphism between the Floer homology groups is induced by an isomorphism that holds at the level of complexes. One consequence of this identification is the independence of $F H_{] a,+\infty[}^{*}(U)$ with respect to $a<0$ when $U$ has restricted contact type boundary.

I know of no similar construction which identifies the homology groups defined in $[\mathrm{CFH}]$ and [V2]. The 1-periodic orbits can no longer be separated in a suitable fashion by the action as in $[\mathrm{He} 2]$ and, for the same truncation by the action, the complex defined in $[\mathrm{CFH}]$ generally involves strictly more orbits than the one in [V2]. The presence of extra generators in the Floer complex raises the probability that "interesting" (i.e. geometric) generators be killed in homology. In this sense the homology defined in [V2] has a more geometric flavour than the one of $[\mathrm{CFH}]$.

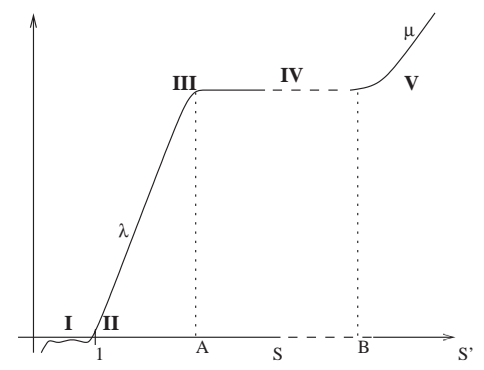

Figure 2: Cofinal family [He2] for the isomorphism [FH1]-[V2]; $S$ and $S^{\prime}$ are the coordinates along the Liouville field of $U$ and along the radial field in $\mathbb{C}^{n}$

One crucial feature of the definitions in $[\mathrm{CFH}, \mathrm{CFHW}]$ is that they are intrinsic and depend only on the interior of the manifold. This will be crucial in proving the stability of the action spectrum (see $\S 4.2$ ). In comparison, the definition in [FH1] is inherently extrinsic, while the one in [V2] is intrinsic but depends on the boundary as well. As a drawback, in the homology of $[\mathrm{CFH}, \mathrm{CFHW}]$ every geometric generator on the boundary appears twice and this may cause some loss of information when computing the global homology. 
Let us remark at this point that it would be interesting to find a version of homology that would depend only on the interior of the manifold and that would use a complex in which every geometric generator enters exactly once. This could lead to topological obstructions for symplectic fillings, as well as to connections with contact homology.

\subsection{Invariance through isotopies}

Floer homology satisfies two important invariance properties, with respect to deformations of the Hamiltonians and with respect to deformations of the symplectic forms. We shall state them precisely below. The Hamitonian invariance is valid under more general hypothesis than the ones adopted here, in particular for some homotopies that are not necessarily increasing. But for our needs the results below will do. Note that it does not matter whether one works with the definition in $[\mathrm{CFH}]$ or with that in [V2].

Theorem 2.1. [V2] Let $H(s), s \in[0,1]$ be an admissible deformation(cf. $\S 1.3)$, such that the 1-periodic orbits of $X_{H(s)}, s \in[0,1]$ all stay in a fixed compact set. Let $a_{s}, b_{s}$ be continuous families of parameters in $\overline{\mathbb{R}}$ such that $H(s)$ has no 1-periodic orbits with action equal to $a_{s}$ or $b_{s}$. There is a canonical isomorphism

$$
F H_{]_{1}, b_{1}\right]}^{*}\left(H_{1}\right) \stackrel{\sim}{\sim} F H_{]_{\left.a_{0}, b_{0}\right]}^{*}}\left(H_{0}\right),
$$

obtained in the usual manner by considering solutions of the equations (1.8 1.9).

One should note that we suppose in particular $H_{0} \prec H_{1}$. We leave it to the reader to extract from the computations given in the next section a ready-made example showing that it is essential to require that all 1-periodic orbits stay in a fixed compact set.

The invariance of Floer homology with respect to deformations of the symplectic form is closely related to the preceding result. It can be given a heuristic interpretation by taking $a_{s} \equiv-\infty, b_{s} \equiv+\infty$ :

Theorem 2.2. [V2] Let $M$ be a compact symplectic manifold with boundary and $\omega_{s}, s \in[0,1]$ an isotopy of symplectic forms with respect to which the boundary $\partial M$ is of contact type. There is an isomorphism $F H^{*}\left(M, \omega_{0}\right) \simeq$ $F H^{*}\left(M, \omega_{1}\right)$ between the nontruncated Floer homologies corresponding to $\omega_{0}$ and $\omega_{1}$.

The isomorphism is again obtained by counting solutions of an equation that is very much similar to (1.8). It is crucial to work with the nontruncated homology in order for the morphism to be well defined. It is also important to note that the isotopy must not necessarily preserve the cohomology class of the symplectic form. Let us also bear in mind the following useful corollary which shows that the nontruncated Floer homology of a manifold with boundary $M$ 
is in fact an invariant of its completion $\widehat{M}$. The result is stated for open sets with smooth boundary only for the sake of applying the above theorem as such, although it is true for general open sets if one suitably defines the Floer homology groups. As a side remark, note that the invariance with respect to deformations of the symplectic form raises the question of the differentiable invariance of Floer homology of manifolds with boundary.

Corollary 2.3. Let $U, U^{\prime}$ be two open sets with smooth boundary in $\widehat{M}$ which satisfy the following conditions:

- $\partial U$ and $\partial U^{\prime}$ are contained in the domain of definition of the Liouville vector field extended to $\widehat{M}$ as $S \frac{\partial}{\partial S}$;

- the Liouville vector field is transverse and outward pointing along $\partial U, \partial U^{\prime}$.

Then

$$
F H^{*}(U) \simeq F H^{*}\left(U^{\prime}\right)
$$

Proof. One can realise a differentiable isotopy between $U$ and $U^{\prime}$ along the Liouville vector field. This corresponds to an isotopy of symplectic forms on $U$ starting from its initial symplectic form $\omega_{0}$ and ending to the one induced from $U^{\prime}$, denoted by $\omega_{1}$. The invariance theorem ensures $F H^{*}\left(U, \omega_{0}\right) \simeq$ $F H^{*}\left(U, \omega_{1}\right)$. But $\left(U, \omega_{1}\right)$ and $\left(U^{\prime}, \omega_{0}\right)$ are symplectomorphic and hence we also have $F H^{*}\left(U, \omega_{1}\right) \simeq F H^{*}\left(U^{\prime}, \omega_{0}\right)$.

\section{A computation: balls in $\mathbb{C}^{n}$}

The Floer cohomology groups $F H_{] a, b]}^{*}$ of the ball $D^{2 n} \subset \mathbb{C}^{n}$ have been computed for the first time in [FHW], together with those of ellipsoids and polydiscs, for arbitrary $a, b \in \overline{\mathbb{R}}$. The nontruncated cohomology groups vanish: $F H^{*}\left(D^{2 n}\right)=$ 0 . The method of $[\mathrm{FHW}]$ consists in approximating the ball by ellipsoids whose characteristics are transversally nondegenerate. The homology of the latter is studied by considering perturbed Hamiltonians whose nontrivial 1-periodic orbits are nondegenerate and come in pairs which correspond to transversally nondegenerate characteristics. The two 1-periodic orbits in a pair correspond to the two critical points of a Morse function defined on the corresponding characteristic, which is identified with $\mathbb{S}^{1}$. The difference between their ConleyZehnder indices is equal to $1=\operatorname{dim} \mathbb{S}^{1}$. The same perturbation technique is used in $[\mathrm{CFHW}]$ in the proof of the stability of the action spectrum of the boundary of a symplectic manifold.

The closed characteristics on the sphere $\mathbb{S}^{2 n-1}=\partial D^{2 n}$ are the great circles together with their positive multiples. They appear in families that are parameterized by the sphere $\mathbb{S}^{2 n-1}$ itself. We shall present in section 3.3 a way of perturbing the natural Hamiltonians $H(z)=h\left(|z|^{2}\right)$ such that every manifold 
of closed characteristics produces two nondegenerate periodic orbits, whose difference of Conley-Zehnder indices is equal to $2 n-1=\operatorname{dim} \mathbb{S}^{2 n-1}$. This is the higher dimensional analogue of the perturbation method in [FHW]. The interest of this point of view lies in that it allows one to understand the vanishing of the Floer homology of the ball without using as an intermediate ingredient the homology of ellipsoids. It also involves some interesting computations of Conley-Zehnder indices and may serve as a hands-on example for the constructions in [Po] $\S 3.4$ and [BPS] $\S 5.2$ which compute the local Floer homology of a Lagrangian intersection and, respectively, of a Hamiltonian admitting a Morse-Bott nondegenerate manifold of 1-periodic orbits.

\subsection{Recollections on the Robbin-Salamon index}

We remind that the Conley-Zehnder index is an integer that is associated to a path of symplectic matrices starting at Id and ending at a matrix $\Psi$ such that $\operatorname{det}(\mathrm{Id}-\Psi) \neq 0$. This is precisely the situation encountered after linearizing the Hamiltonian flow along a nondegenerate periodic orbit.

We shall compute below some Conley-Zehnder indices through the intermediate of the Robbin-Salamon index [RS1]. The latter generalizes the ConleyZehnder index to arbitrary paths in $\operatorname{Sp}(2 n, \mathbb{R})$. The reader can consult [S2] §2.4. for a review of the basic properties of the Conley-Zehnder index and for its computation using crossing numbers, as well as [RS1] for a complete account on the Robbin-Salamon index. We shall sketch here only the facts that are used in the sequel.

The Robbin-Salamon index $i_{R S}(\Psi)$ of an arbitrary path of symplectic matrices $\Psi:[0,1] \longrightarrow \operatorname{Sp}(2 n)$ is defined as the index of the Lagrangian path $\operatorname{gr}(\Psi)$ in $\left(\mathbb{R}^{2 n} \times \mathbb{R}^{2 n},(-\omega) \oplus \omega\right)$ relative to the diagonal $\Delta=\left\{(X, X): X \in \mathbb{R}^{2 n}\right\}$. Its computation makes use of the notions of simple crossing and crossing form.

A crossing is a number $t \in[0,1]$ such that $\operatorname{gr} \Psi(t) \cap \Delta \neq 0$ or else $\operatorname{ker}(\mathrm{Id}-$ $\Psi(t)) \neq 0$. The crossing form at a crossing $t_{0}$ is the quadratic form $\Gamma\left(\Psi, t_{0}\right)$ : $\operatorname{ker}(\mathrm{Id}-\Psi(t)) \longrightarrow \mathbb{R}$ defined by

$$
\Gamma\left(\Psi, t_{0}\right)(v)=\omega_{0}\left(v, \dot{\Psi}\left(t_{0}\right) v\right) .
$$

Any path in $\operatorname{Sp}(2 n, \mathbb{R})$ is solution to a differential equation $\dot{\Psi}(t)=J_{0} S(t) \Psi(t)$, with $S(t)$ a symmetric matrix. One can therefore write

$$
\Gamma\left(\Psi, t_{0}\right)(v)=\left\langle v, S\left(t_{0}\right) v\right\rangle .
$$

A crossing $t_{0}$ is called simple if the crossing form $\Gamma\left(\Psi, t_{0}\right)$ is nondegenerate. A simple crossing is necessarily isolated.

Any path $\Psi$ is homotopic with fixed end points to a path having only simple crossings. The index $i_{R S}(\Psi)$ of a path having only simple crossings is defined to be

$$
i_{R S}(\Psi)=\frac{1}{2} \operatorname{sign} \Gamma(\Psi, 0)+\sum_{0<t<1} \operatorname{sign} \Gamma(\Psi, t)+\frac{1}{2} \operatorname{sign} \Gamma(\Psi, 1),
$$


where the summation runs over all simple crossings of $\Psi$. We recall that the signature of a nondegenerate quadratic form is the difference between the number of its positive eigenvalues and the number of its negative eigenvalues.

The main features of the Robbin-Salamon index are the following.

i. additivity under concatenations of paths: $i_{R S}\left(\left.\Psi\right|_{[a, b]}\right)+i_{R S}\left(\left.\Psi\right|_{[b, c]}\right)=$ $i_{R S}\left(\left.\Psi\right|_{[a, c]}\right)$

ii. $i_{R S}$ characterizes paths up to homotopy with fixed end points ;

iii. additivity under products: $i_{R S}\left(\Psi^{\prime} \oplus \Psi^{\prime \prime}\right)=i_{R S}\left(\Psi^{\prime}\right)+i_{R S}\left(\Psi^{\prime \prime}\right)$.

\subsection{Direct computation of the cohomology of a ball}

The argument that we present below appears in [FHW] and [V2]. Let us identify $\mathbb{C}^{n}$ with $\mathbb{R}^{2 n}$ by associating to $z=x+i y, x, y \in \mathbb{R}^{n}$ the vector $\left(x_{1}, \ldots, x_{n}, y_{1}, \ldots, y_{n}\right)$. We consider on $\mathbb{C}^{n}$ the standard symplectic form $\omega_{0}=\sum_{i=1}^{n} d x_{i} \wedge d y_{i}$ with its primitive $\lambda_{0}=\frac{1}{2} \sum_{i=1}^{n} x_{i} d y_{i}-y_{i} d x_{i}$. The associated Liouville vector field $X(z)=\frac{1}{2} z, z \in \mathbb{C}^{n}$ is transverse to all the spheres that are centered at the origin. We shall use the standard almost complex structure given by complex multiplication with $i$ i.e. $J\left(x_{1}, \ldots, x_{n}, y_{1}, \ldots, y_{n}\right)=$ $\left(-y_{1}, \ldots,-y_{n}, x_{1}, \ldots, x_{n}\right)$. The Reeb vector field on $\mathbb{S}^{2 n-1}$ is $X_{\text {Reeb }}(z)=2 J z$ and its closed orbits are the great circles and their positive multiples, with action $k \pi, k \in \mathbb{N}^{*}$. It is clearly possible to construct a cofinal family of Hamiltonians of the form $H_{\lambda}(z)=\rho\left(|z|^{2}\right)$, with $\rho:\left[0, \infty\left[\longrightarrow \mathbb{R}\right.\right.$ satisfying $\left.\rho\right|_{[0,1]}<0$, $\rho^{\prime \prime} \geq 0,\left.\rho^{\prime}\right|_{[1+\epsilon, \infty[}=\lambda,\left.\rho^{\prime}\right|_{[0,1+\epsilon)}>0, \epsilon=\epsilon(\lambda)>0$. When $\lambda>0$ is not a multiple of $\pi$ the 1-periodic orbits of $H_{\lambda}$ are parameterized by the critical point 0 (the constant orbit) and the spheres $\left\{z: \rho^{\prime}\left(|z|^{2}\right)=l \pi\right\}, 1 \leq l \leq k$ where $k \pi<\lambda<(k+1) \pi$.

Let therefore $\lambda \neq k \pi$ and take $b$ sufficiently large compared to $\lambda$. We claim that

$$
F H_{]-\infty, b]}^{*}\left(H_{\lambda}\right)=F H_{]-\infty, b]}^{*}\left(\lambda|z|^{2}-c\right),
$$

where $0<c \leq 1$ is a constant such that $\lambda|z|^{2}-c \leq H_{\lambda}$. Note that it is always possible to choose $c \leq 1$ if $\lambda$ is large enough, due to the cofinality of the family $\left(H_{\lambda}\right)_{\lambda}$. We can connect $\lambda|z|^{2}-c$ with $H_{\lambda}$ through an admissible homotopy of Hamiltonians of the same type as $H_{\lambda}$. The periodic orbits that are created correspond to closed characteristics on $\mathbb{S}^{2 n-1}$ and we easily see that their action will not cross $b$ if the latter is large enough (e.g. $b>\lambda+1$ for our choice of $c$ ). The isotopy invariance theorem 2.1 implies (3.1).

In order to fix ideas, let us suppose that $k \pi<\lambda<(k+1) \pi$. The Hamiltonian $Q_{\lambda}=\lambda|z|^{2}-c$ has a unique 1-periodic orbit: the constant $z=0$. We have seen above that the natural grading on Floer (co)homology is given by the ConleyZehnder index taken with opposite sign. This amounts to the computation of the index of the 1-periodic orbits of the vector field $-X_{Q_{\lambda}}(z)=2 \lambda J z$, whose flow is $\varphi_{t}(z)=e^{2 i \lambda t} z$. Its linearization is $d \varphi_{t}(z) \cdot Y=e^{2 i \lambda t} Y$, or 


$$
d \varphi_{t}=\left(\begin{array}{ccc}
\left(\begin{array}{cc}
\cos 2 \lambda t & -\sin 2 \lambda t \\
\sin 2 \lambda t & \cos 2 \lambda t
\end{array}\right) & & 0 \\
& & \ddots \\
0 & & \left(\begin{array}{cc}
\cos 2 \lambda t & -\sin 2 \lambda t \\
\sin 2 \lambda t & \cos 2 \lambda t
\end{array}\right)
\end{array}\right)
$$

The Conley-Zehnder index is $i_{C Z}\left(d \varphi_{t}\right)=n \cdot i_{C Z}\left(\left(\begin{array}{cc}\cos 2 \lambda t & -\sin 2 \lambda t \\ \sin 2 \lambda t & \cos 2 \lambda t\end{array}\right)\right)=$ $n(2 k+1)$ : the path $t \mapsto e^{2 i \lambda t}$ in $\operatorname{Sp}(2, \mathbb{R})$ has $k$ interior crossings at $t=l \pi / \lambda$, $1 \leq l \leq k$ and one initial crossing at $t=0$, with an intersection form of constant signature equal to 2 . We get thus the nontruncated cohomology

$$
F H^{*}\left(H_{\lambda}\right)=\left\{\begin{array}{cl}
\mathbb{Z}, & *=n(2 k+1), \\
0, & * \neq n(2 k+1)
\end{array}\right.
$$

As a consequence we have

$$
F H^{*}\left(D^{2 n}\right)=\lim _{\longleftarrow} F H^{*}\left(H_{\lambda}\right)=0 .
$$

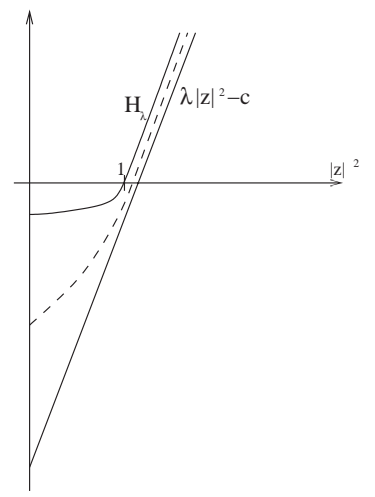

Figure 3: Computation of the Floer homology of $D^{2 n}$

\subsection{Alternative point of view: perturbation of critical Morse-Bott manifolds}

We present now an alternative point of view which consists in directly perturbing the critical manifolds of $H_{\lambda}$. Our computation concerns the (opposite) 
Conley-Zehnder indices of the corresponding generators in the Floer complex.

Let us first compute the Robbin-Salamon indices $i_{R S}$ for the 1-periodic orbits of $H_{\lambda}, k \pi<\lambda<(k+1) \pi$ considered with the opposite orientation. We have $-X_{H_{\lambda}}(z)=2 \rho^{\prime}\left(|z|^{2}\right) J z$ and the flow is $\varphi_{t}(z)=e^{2 \rho^{\prime}\left(|z|^{2}\right) J t} z$. The linearization of the flow is

$$
d \varphi_{t}(z) \cdot Y=e^{2 \rho^{\prime}\left(|z|^{2}\right) J t} \cdot Y+2 \rho^{\prime \prime}\left(|z|^{2}\right) J t \cdot e^{2 \rho^{\prime}\left(|z|^{2}\right) J t} \cdot 2\langle z, Y\rangle \cdot z
$$

For $z=0$ the linearization is $d \varphi_{t}(0)=e^{2 \rho^{\prime}(0) J t}$ and satisfies the differential equation $\dot{\Psi}(t)=J \operatorname{diag}\left(2 \rho^{\prime}(0)\right) \Psi(t), \Psi(0)=$ Id. For a sufficiently large $\lambda$ the cofinality of $H_{\lambda}$ imposes $\rho^{\prime}(0)<\pi$. As a consequence the path $e^{2 \rho^{\prime}(0) J t}$ has a unique crossing at $t=0$ with intersection form of signature $2 n$. We get

$$
i_{R S}(z \equiv 0)=n .
$$

Let us now look at a sphere $S_{l}=\left\{z: \rho^{\prime}\left(|z|^{2}\right)=l \pi\right\}, 1 \leq l \leq k$. Consider the $J$-invariant symplectic decomposition of $T_{z} \mathbb{R}^{2 n}, z \in S_{l}$ given by

$$
T_{z} \mathbb{R}^{2 n}=\mathbb{R}\left\langle\frac{1}{2 l \pi|z|^{2}} z\right\rangle \oplus \mathbb{R}\langle 2 l \pi J z\rangle \oplus \xi_{z},
$$

where $\xi=T S_{l} \cap J T S_{l}$ is the contact distribution on $S_{l}$. The matrix of $d \varphi_{t}$ is written with respect to this decomposition as

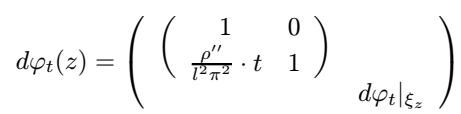

As $\left.d \varphi_{t}(z)\right|_{T_{z} S_{l}}=e^{2 l \pi J t}$ we can still write in the canonical trivialization of $T \mathbb{R}^{2 n}$ :

$$
d \varphi_{t}(z)=\chi(t) \circ \Psi(t)
$$

with $\Psi(t)=e^{2 l \pi J t}$ and

$$
\left.\chi(t)=\left(\begin{array}{cc}
1 & 0 \\
\frac{\rho^{\prime \prime}}{l^{2} \pi^{2}} \cdot t & 1
\end{array}\right) \quad \begin{array}{l} 
\\
\operatorname{Id}_{2 n-2}
\end{array}\right)
$$

The homotopy (cf. [CFHW])

$$
K(s, t)= \begin{cases}\chi(s t) \Psi\left(\frac{2 t}{s+1}\right), & t \leq \frac{s+1}{2} \\ \chi((s+2) t-(s+1)) \Psi(1), & \frac{s+1}{2} \leq t\end{cases}
$$

connects with fixed ends the path $\chi(t) \Psi(t)$ to the concatenation of $\Psi(t)$ and $\chi(t) \Psi(1)$. We thus get

$$
i_{R S}\left(d \varphi_{t}(z)\right)=i_{R S}(\Psi(t))+i_{R S}(\chi(t) \Psi(1))=2 \ln +\frac{1}{2}
$$


or else

$$
i_{R S}\left(S_{l}\right)=2 \ln +\frac{1}{2} .
$$

Once we have computed the indices before perturbing, we can go on with the description of the latter. By a time-dependent change of variables we can suppose that

$$
X_{H_{t}} \equiv 0 \quad \text { on } \quad S_{l} .
$$

We leave it to the reader to work it out or check [CFHW], p.34. The idea is to spin $\mathbb{S}^{l}$ the other way round so that all its points become fixed under the new flow.

Let us now choose a Morse function having exactly two critical points $h$ : $S_{l} \longrightarrow \mathbb{R}$. We extend $h$ to $\widetilde{h}$ in a neighbourhood of $S_{l}$ by $\widetilde{h}(z, S)=h(z)$, where $(z, S) \longmapsto S z$ is an orthogonal parameterization of a neighbourhood of $S_{l}$ by $\left.S_{l} \times\right] 1-\epsilon, 1+\epsilon\left[\right.$. Let $H_{\delta}=H+\delta \tilde{h}, \delta>0$. One can show by an argument similar to [CFHW] that, for $\delta$ small enough, $X_{H_{\delta}}$ has precisely two 1-periodic (constant) orbits in the neighbourhood of $S_{l}$, that correspond to the two critical points of $h$. Let $\Psi(t)$ be the linearized flow of $-X_{H}, \Phi_{\delta}(t)$ be the linearized flow of $-X_{\delta \widetilde{h}}, \Psi_{\delta}(t)$ be the linearized flow of $-X_{H_{\delta}}$. For small $\delta$ the paths $\Psi_{\delta}(t)$ and $\Psi(t) \Phi_{\delta}(t)$ are homotopic with ends in $\operatorname{Sp}_{0}(2 n, \mathbb{R})$, the set of symplectic matrices with eigenvalues different from 1 . The homotopy is given by $L(s, t)=\Psi_{s \delta}(t) \Phi_{(1-s) \delta}(t)$. The same argument as above shows that

$$
i_{R S}\left(\Psi_{\delta}\right)=i_{R S}(\Psi)+i_{R S}\left(\Psi(1) \Phi_{\delta}\right) .
$$

But $i_{R S}(\Psi)=\frac{1}{2}+2 \ln$ by the above computations. It is therefore enough to find the index of $M(t)=\Psi(1) \Phi_{\delta}(t)$ and we again use the definition. Then $-X_{\delta \widetilde{h}}(z)=\delta J \nabla \widetilde{h}(z)=\delta J \nabla h(z), z \in S_{l}$ and the linearization of the flow at the critical point $z_{0} \in S_{l}$ is a solution of the differential equation $\dot{A}(t)=$ $D\left(-X_{\delta \widetilde{h}}\left(z_{0}\right)\right) \cdot A(t), A(0)=\operatorname{Id}_{2 n}$. This implies

$$
d \varphi_{t}^{-X_{\delta \tilde{h}}}\left(z_{0}\right)=e^{\delta t J \nabla^{2} \widetilde{h}\left(z_{0}\right)} .
$$

But it is easy to see that $\nabla^{2} \widetilde{h}\left(z_{0}\right)=\left(\begin{array}{cc}\nabla^{2} h\left(z_{0}\right) & 0 \\ 0 & 0\end{array}\right)$ in the decomposition $T_{z_{0}} \mathbb{R}^{2 n}=T_{z_{0}} S_{l} \oplus \mathbb{R}\left\langle z_{0}\right\rangle$. This immediately implies $\operatorname{ker}\left(\operatorname{Id}-\Psi(1) d \varphi_{t}^{-X_{\delta \tilde{h}}}\right)=\{0\}$ for $t>0$ and one is reduced to compute the intersection form at $t=0$. We have

and therefore

$$
\operatorname{ker}\left(\operatorname{Id}-\Psi(1) \Phi_{\delta}(0)\right)=T_{z_{0}} S_{l}
$$

$$
\operatorname{sign} \Gamma\left(\Phi_{\delta}, 0\right)=\operatorname{sign} \delta \nabla^{2} h\left(z_{0}\right) .
$$

The signature of $\Gamma$ is therefore equal to $2 n-1$ at the critical point of index 0 , respectively equal to $-(2 n-1)$ at the critical point of index $2 n-1$. This means 


$$
\begin{aligned}
& i_{R S}\left(\Psi(1) \Phi_{\delta}(t)\right)= \pm\left(n-\frac{1}{2}\right) \text { and } \\
& i_{R S}\left(d \varphi_{t}^{-X_{H_{\delta}}}\left(z_{0}\right)\right)= \begin{cases}2 l n+n, & \operatorname{ind}_{\text {Morse }}\left(z_{0}\right)=0 \\
2 l n-n+1, & \operatorname{ind}_{\text {Morse }}\left(z_{0}\right)=2 n-1\end{cases}
\end{aligned}
$$

The situation is therefore the following: for $k \pi<\lambda<(k+1) \pi$ the Hamiltonian $H_{\lambda}$ admits a constant orbit $z \equiv 0$ of index $n$ and $k$ manifolds of periodic orbits diffeomorphic to $\mathbb{S}^{2 n-1}$ and having indices $2 l n+\frac{1}{2}, 1 \leq l \leq k$. After perturbation, each such manifold produces two nondegenerate orbits of indices $2 \ln -n+1$ and $2 \ln +n$, corresponding respectively to the maximum and minimum of the perturbing function. The truncated homology $F H_{-\infty, b]}^{*}\left(H_{\lambda}\right), b>\lambda$ was already computed above and it is nontrivial only in degree $n+2 k n$, where it equals $\mathbb{Z}$. We infer that the arrows in the perturbed Floer complex run as shown below, where the third line indicates the degree in the Floer complex, given by the opposite of the Conley-Zehnder index and the first line indicates the action of the corresponding orbit (compare with [FHW]).

$$
\begin{aligned}
& \epsilon \quad \pi-\epsilon \quad \pi+\epsilon \quad 2 \pi-\epsilon \quad(k-1) \pi-\epsilon \quad(k-1) \pi+\epsilon \quad k \pi-\epsilon
\end{aligned}
$$

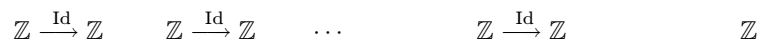

$$
\begin{aligned}
& n \quad n+1 \quad 3 n \quad 3 n+1 \quad(2 k-1) n \quad(2 k-1) n+1 \quad(2 k+1) n
\end{aligned}
$$

This allows one to also compute the values of the truncated Floer cohomology groups $F H_{] a, b]}^{*}\left(D^{2 n}\right)$ for arbitrary $a, b \in \overline{\mathbb{R}}$, recovering the results in [FHW].

\section{Applications}

As we have already pointed out, each of the three constructions described above has specific features that allow particular applications. We shall present below the symplectic classification of ellipsoids and polydiscs using symplectic homology as in [FH1], the stability of the action spectrum of the contact type boundary of symplectic manifolds [CFHW], as well as applications to Weinstein's conjecture and exact Lagrange embeddings [V2]. These problems will make the reader familiar with some techniques that are representative for the field.

\subsection{Polydiscs and ellipsoids}

We use in this section the homology groups defined by Floer and Hofer or those defined by Viterbo (cf. sections 1.3, 1.3, 2.2). We explain the following two theorems. 
Theorem 4.1. Let $r=\left(r_{1}, r_{2}, \ldots, r_{n}\right) \in \mathbb{R}_{+}^{*}, r_{1} \leq r_{2} \leq \ldots \leq r_{n}$ and denote

$$
E(r)=\left\{\left(z_{1}, \ldots, z_{n}\right) \in \mathbb{C}^{n}: \sum_{i=1}^{n} \frac{\left|z_{i}\right|^{2}}{r_{i}^{2}}<1\right\} .
$$

Then $E(r)$ and $E\left(r^{\prime}\right)$ are symplectically diffeomorphic if and only if $r=r^{\prime}$.

Theorem 4.2. Let $r=\left(r_{1}, r_{2}, \ldots, r_{n}\right) \in \mathbb{R}_{+}^{*}, r_{1} \leq r_{2} \leq \ldots \leq r_{n}$ and denote

$$
D^{2 n}(r)=B^{2}\left(r_{1}\right) \times \ldots \times B^{2}\left(r_{n}\right) \subset \mathbb{C}^{n} .
$$

Then $D^{2 n}(r)$ and $D^{2 n}\left(r^{\prime}\right)$ are symplectically diffeomorphic if and only if $r=r^{\prime}$.

Both theorems follow from the explicit computation of the truncated symplectic (co)homology of ellipsoids and polydiscs and we shall address the case of ellipsoids. The computation for polydiscs is of a similar nature and we refer the interested reader to $[\mathrm{FHW}]$, p. 583.

Sketch of proof for Theorem 4.1. One interesting remark concerning the Floer complex that we have computed in $\S 3$ is that the same homology would have been obtained out of the following complex:

$$
\begin{gathered}
0 \longrightarrow(\mathbb{Z}, n ; 0) \stackrel{\mathrm{Id}}{\longrightarrow}(\mathbb{Z}, n+1 ; \pi) \stackrel{0}{\longrightarrow}(\mathbb{Z}, n+2 ; \pi) \stackrel{\mathrm{Id}}{\longrightarrow} \cdots \stackrel{0}{\longrightarrow}(\mathbb{Z}, 3 n ; \pi) \\
\stackrel{\mathrm{Id}}{\longrightarrow}(\mathbb{Z}, 3 n+1 ; 2 \pi) \stackrel{0}{\longrightarrow} \cdots
\end{gathered}
$$

The meaning of the notation is the following: in a term $(\mathbb{Z}, k ; \alpha)$ we have $k$ standing for the grading and $\alpha$ for the corresponding action of the generator. If one wishes to compute the truncated cohomology $F H_{] a, b]}^{*}$ the complex to consider is $C_{b} / C_{a}$, with $C_{b}=\oplus_{\alpha \leq b}(\mathbb{Z}, k ; \alpha)$. This corresponds to formally replacing the arbitrarily small $\epsilon$ in (3.4) with 0 .

The complex (4.1) is precisely the one used in [FHW] and arises in a natural geometric way. To the difference of our method of perturbing the spheres of characteristics with action $k \pi, k \in \mathbb{Z}_{+}^{*}$ and produce 2 orbits, the method of the original paper is to approximate the ball by a generic ellipsoid having $n$ simple characteristics $\left(0, \ldots, r_{j} e^{2 \pi i t / r_{j}}, \ldots, 0\right)$ whose areas $\left\{\pi r_{j}^{2}\right\}, 1 \leq j \leq n$ are linearly independent over $\mathbb{Q}$, and then perturb each such characteristic in order to finally produce $2 n$ nondegenerate orbits. For the perturbations of the simple characteristics that arise for an approximation of the sphere the indices are precisely $n+1, n+2, \ldots, 3 n$.

This discussion is of course no proof of the general case, but gives a geometric explanation for the construction of the complex that computes the symplectic cohomology of a general ellipsoid, where every closed characteristic (simple or not) gives rise to 2 orbits having action arbitrarily close to some $k \pi r_{j}^{2}, k \in \mathbb{Z}_{+}^{*}$.

$$
\begin{gathered}
F H_{] a, b]}^{*}(E(r))=H^{*}\left(C_{b} / C_{a}\right), \\
C_{b}=0 \longrightarrow(\mathbb{Z}, n) \stackrel{\text { Id }}{\longrightarrow}(\mathbb{Z}, n+1) \stackrel{0}{\longrightarrow}(\mathbb{Z}, n+2) \stackrel{\text { Id }}{\longrightarrow} \cdots \stackrel{0}{\longrightarrow}(\mathbb{Z}, n+2 m(b ; r)) \\
\longrightarrow 0 \longrightarrow 0 \longrightarrow \cdots
\end{gathered}
$$


where

$$
m(b ; r)=\#\left\{(k, j) \in \mathbb{Z}_{+}^{*} \times\{1, \ldots, n\}: k \pi r_{j}^{2} \leq b\right\} .
$$

The reader can easily get convinced that, for the ball of radius one, the two complexes (4.1) and (4.2) are the same.

In the case of a generic ellipsoid Theorem 4.1 follows now at once, as one can easily compute for example $F H_{\left.] \pi r_{j}^{2}-\epsilon, \pi r_{j}^{2}+\epsilon\right]}^{n+2 j} \simeq \mathbb{Z}$ and $F H_{\left.] \pi r_{j}^{2}-\epsilon, \pi r_{j}^{2}+\epsilon\right]}^{*}=0$ if $* \neq n+2 j$.

\subsection{Stability of the action spectrum}

In this section we use the version of symplectic homology described in [CFHW] (cf. section 1.3). The "stability of the action spectrum" is a problem related to the question of the extent to which the interior of a symplectic manifold determines its boundary. One answer is that, in the nondegenerate case, the set of values of the areas of closed characteristics on the boundary is determined by the interior of the manifold. We first introduce the relevant definitions.

Definition 4.3. A symplectic manifold $(M, \omega)$ is said to satisfy the symplectic asphericity condition if $\left\langle\omega, \pi_{2}(M)\right\rangle=0$. If in addition $\left\langle c_{1}(M), \pi_{2}(M)\right\rangle=0$ we say that the manifold is strongly symplectically aspherical (the Chern class is computed with respect to an almost complex structure $J$ that is compatible with the symplectic form, and does not depend on $J$ ).

Definition 4.4. Let $(M, \omega)$ be a compact symplectic manifold with nonempty contact type boundary. Assume $(M, \omega)$ is strongly symplectically aspherical. We note $X$ the Liouville vector field, $\lambda$ the Liouville form and $X_{\text {Reeb }}$ the Reeb vector field.

Let $x:[0, T] \longrightarrow \partial M$ be a contractible closed characteristic with $x(0)=x(T)$. We define the action of $x$ to be

$$
A(x)=\int_{0}^{T} x^{*} \lambda .
$$

We define the index $i_{R S}(x)$ of $x$ to be the Robbin-Salamon index of the path

$$
\Gamma(t)=\Psi\left(e^{2 \pi i t / T}\right) \circ \psi_{t_{*}} \in \operatorname{Symp}\left(T_{x(0)} M\right),
$$

where $\psi_{t}$ is the flow of the Reeb vector field on $\partial M, \psi_{t_{*}}: T_{x(0)} M \longrightarrow T_{x(t)} M$ denotes its linearization extended by $\psi_{t_{*}}\left(X(x(0))=X(x(t))\right.$, and $\Psi:\left.T M\right|_{D^{2}} \longrightarrow$ $D^{2} \times T_{x(0)} M$ denotes a trivialization of TM over a filling disc $D^{2}$ for $x$.

One should note that the condition $\left\langle\omega, \pi_{2}(M)\right\rangle=0$ ensures that the value of the action is independent on the Liouville form, as we have $\int_{0}^{T} x^{*} \lambda=\int_{D^{2}} \bar{x}^{*} \omega$, with $\bar{x}: D^{2} \longrightarrow M$ such that $\bar{x}\left(e^{2 \pi i t / T}\right)=x(t)$. On the other hand, the hypothesis $\left\langle c_{1}, \pi_{2}(M)\right\rangle=0$ ensures that any two trivializations of $T M$ over disks with common boundary are homotopic along the boundary and this means that the value of $i_{R S}(x)$ does not depend on the choice of trivialization. 
Definition 4.5. $[C F H W]$ Let $(M, \omega)$ be a compact symplectic manifold with nonempty contact type boundary, satisfying the strong symplectic asphericity condition $\left\langle\omega, \pi_{2}(M)\right\rangle=\left\langle c_{1}, \pi_{2}(M)\right\rangle=0$. Denote by $\mathcal{C}(M)$ the set of contractible closed characteristics on $\partial M$. The action spectrum $\mathcal{A}(\partial M)$ is defined as

$$
\mathcal{A}(\partial M)=\left\{\left(A(x),-i_{R S}(x)\right) \mid x \in \mathcal{C}(M)\right\}
$$

Definition 4.6. Let $x:[0, T] \longrightarrow \partial M$ be a closed characteristic of period $T$, parameterized by the Reeb vector field. We say that $x$ is transversally nondegenerate if the restriction of the linearization of the Reeb flow

$$
\psi_{T_{*}}: \xi_{x(0)} \longrightarrow \xi_{x(0)}
$$

has no eigenvalue equal to 1.

We will see below that, under this hypothesis, the index $i_{R S}(x)$ is an integer.

Theorem 4.7. [CFHW] Let $M$ and $N$ be compact symplectic manifolds with nonempty boundary of contact type, satisfying the symplectic asphericity condition and having transversally nondegenerate closed characteristics. Assume that their interiors $\dot{M}, \dot{N}$ are symplectically diffeomorphic. Then

$$
\mathcal{A}(\partial M)=\mathcal{A}(\partial N) \text {. }
$$

This is an immediate consequence of the result below, where the Floer homology groups are defined according to [CFHW]. Indeed, this definition of the Floer homology groups for a manifold $M$ depends only on the interior $\dot{M}$ (the admissible Hamiltonians have compact support in $\dot{M}$ ) and on the possibility to "complete" $\dot{M}$ by adding a boundary of contact type.

Theorem 4.8. Let $M$ be a manifold satisfying the hypotheses of Theorem 4.7 and let $a \in \mathbb{R}^{*}$.

a) The groups $F H_{\mid a-\epsilon, a+\epsilon]}^{*}(M)$ become independent of $\epsilon>0$ as soon as the latter is sufficiently small. We denote them by $F H_{a}^{*}(M)$.

b) If $(a, k) \notin \mathcal{A}(\partial M)$ for all $k \in \mathbb{Z}$ then $F H_{a}^{k}(M)=0$. If $(a, k) \in \mathcal{A}(\partial M)$ has multiplicity $n$, then it gives rise to $n$ copies of $\mathbb{Z}$ as direct summands in $F H_{a}^{k}(M)$ and to $n$ copies of $\mathbb{Z}$ as direct summands in $F H_{a}^{k+1}(M)$.

We note that the hypothesis $a \neq 0$ is necessary for the groups $F H_{] a-\epsilon, a+\epsilon]}^{*}(M)$ to be well defined (cf. $\S 1.3)$.

Sketch of proof. The nondegeneracy hypothesis implies that the characteristics have geometrically isolated images. In particular, the values of their action form a discrete set $\mathcal{A} \subset \mathbb{R}$. If $a \in \mathcal{A}$ then it is possible to choose $\epsilon>0$ such that ]$a-\epsilon, a+\epsilon] \cap \mathcal{A}=\{a\}$ and the invariance property of symplectic homology ensures that all $F H_{\left.] a-\epsilon^{\prime}, a+\epsilon^{\prime}\right]}^{*}(M)$ are isomorphic for $0<\epsilon^{\prime} \leq \epsilon$. The same 
holds if $a \notin \mathcal{A}$, with the additional information that this group is now equal to zero by definition. This proves a) and the first assertion in b).

Let us now focus on the second assertion in b). The Liouville flow gives rise to the usual trivialization of a neighbourhood of the boundary as $\partial M \times[1-\delta, 1]$, $\delta>0$ sufficiently small. Denote $S$ the coordinate on the second factor. One considers a cofinal family of Hamiltonians (Figure 4) which verify $H(p, S)=$ $h(S)$ on $\partial M \times[1-\delta, 1]$, with $h \leq 0$ increasing, $h \equiv$ ct. on $\left[1-\delta, r_{1}\right], h$ strictly convex on $\left[r_{1}, r_{2}\right], h^{\prime} \equiv$ ct. on $\left[r_{2}, r_{3}\right], h$ strictly concave on $\left[r_{3}, r_{4}\right], h \equiv 0$ on $\left[r_{4}, 1\right]$. Here $1-\delta<r_{1}<r_{2}<r_{3}<r_{4}<1$ and $r_{1} \longrightarrow 1$ as $H \stackrel{\prec}{\longrightarrow}-\infty$. Moreover, one assumes that the value of $h^{\prime}$ on $\left[r_{2}, r_{3}\right]$ is not equal to the action of any closed characteristic on $\partial M$.

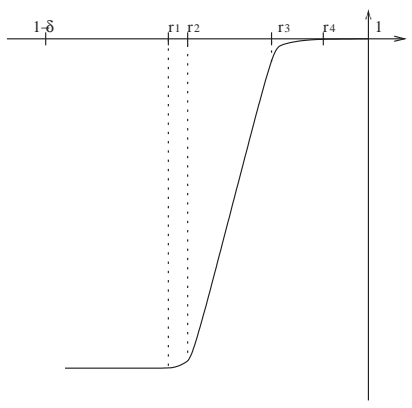

Figure 4: Cofinal family in the proof of the stability of the action spectrum

These Hamiltonians have highly degenerate 1-periodic orbits but the ones having nonzero action are made nondegenerate by an arbitrarily small perturbation away from $\partial M \times\left[r_{4}, 1\right]$. After perturbing, the 1-periodic orbits of $H$ fall into the following classes:

- constants in $\partial M \times\left[r_{4}, 1\right]$ with action 0 ;

- isolated constants away from $\partial M \times[1-\delta, 1]$, with action approximately equal to $-\min H \longrightarrow+\infty$;

- every characteristic $x$ with action less than $\max h^{\prime}$ is seen twice in the unperturbed Hamiltonian: once in $\partial M \times\left[r_{1}, r_{2}\right]$ (call the corresponding orbit $x_{1}$ ) and once in $\partial M \times\left[r_{3}, r_{4}\right]$ (call the corresponding orbit $x_{2}$ ). After perturbation, each of these produces two nondegenerate periodic orbits $x_{1}^{ \pm}, x_{2}^{ \pm}$. Moreover $A_{H}\left(x_{1}^{ \pm}\right) \longrightarrow+\infty, A_{H}\left(x_{2}^{ \pm}\right) \longrightarrow A(x)$.

A computation of the Robbin-Salamon indices very similar in spirit to the 
one already performed in $\S 3$ shows that

$$
i_{R S}\left(x_{1}\right)=i_{R S}(x)+\frac{1}{2}, \quad i_{R S}\left(x_{2}\right)=i_{R S}(x)-\frac{1}{2},
$$

while

$$
i_{R S}\left(x_{i}^{ \pm}\right)=i_{R S}\left(x_{i}\right) \mp \frac{1}{2}, \quad i=1,2 .
$$

In the limit the only orbits whose action belongs to $[A(x)-\epsilon, A(x)+\epsilon]$ are $x_{2}^{ \pm}$, with indices $i_{R S}\left(x_{2}^{-}\right)=i_{R S}(x), i_{R S}\left(x_{2}^{+}\right)=i_{R S}(x)-1$ or, otherwise stated

$$
-i_{R S}\left(x_{2}^{-}\right)=-i_{R S}(x), \quad-i_{R S}\left(x_{2}^{+}\right)=-i_{R S}(x)+1 .
$$

If, for a given value $a$ of the action, there is a single characteristic taking on this value, then the computation of $F H_{]_{a-\epsilon, a+\epsilon]}^{*}}(H)$ is reduced to the understanding of the differential in the Floer complex $\mathbb{Z}\left\langle x_{2}^{-}\right\rangle \longrightarrow \mathbb{Z}\left\langle x_{2}^{+}\right\rangle$. We have seen in $\S 3$ as a by-product of the direct calculation of the Floer cohomology that the differential between the perturbed images of a sphere of periodic orbits is zero. A direct argument based on the implicit function theorem and the isotopy invariance of Floer cohomology can be applied in the present situation to yield the same result, and generalize it to the case where there are several closed characteristics with action $a$.

\subsection{Applications to Weinstein's conjecture and exact La- grange embeddings}

In this section we use the Floer cohomology groups as defined by Viterbo (cf. section 1.3). We have already mentioned that they are useful for proving the existence of closed characteristics on contact type hypersurfaces. The first key tool is provided by the map

$$
F H^{*}(M) \stackrel{c^{*}}{\longrightarrow} H^{n+*}(M, \partial M), \quad n=\frac{1}{2} \operatorname{dim} M
$$

obtained by restricting the range of the action and by using the isomorphism

$$
F H_{] a, 0^{+}\right]}^{*}(M) \simeq H^{n+*}(M, \partial M), \quad a<0 .
$$

Here $0^{+}$stands for a small enough positive number. As we have already pointed out in $\S 1.2$, when restricting the range of the action and considering autonomous Hamiltonians $H$ that are sufficiently small in the $C^{2}$ norm, the Floer complex reduces to the Morse complex of the vector field $\nabla H$. By construction, the latter is outward pointing along $\partial M$. We get in this way the homology of $M$ relative to the boundary, graded by the Morse index of the critical points of $-H$, the function for which $\nabla H$ is negative pseudo-gradient (see also [O], Ch. 3 for further details on this point). 
By the very construction of Floer homology we see that the failure of $c^{*}$ to be bijective implies the existence of a closed characteristic on $\partial M$, which corresponds to the appearance in the Floer complex of a generator other than a critical point of a Morse function defined on $\operatorname{int}(M)$. We follow [V2] and consider the following definition.

Definition 4.9. [V2] A manifold $M$ satisfies the algebraic Weinstein conjecture $(A W C)$ if there is a ring of coefficients such that the map $F H^{*}(M) \stackrel{c^{*}}{\longrightarrow}$ $H^{n+*}(M, \partial M)$ is not an isomorphism.

It is clear that AWC implies the validity of Weinstein's conjecture for $\partial M$. In certain situations that we are going to state below, the AWC property is inherited by codimension 0 submanifolds in $M$ and it will prove useful to distinguish between the situations where the morphism $F H^{*}(M) \longrightarrow H^{n+*}(M, \partial M)$ fails to be injective or fails to be surjective (we shall then say that $M$ satisfies case a), respectively b) of the AWC property).

The second key tool is the existence [V2] $\$ 2$ of a transfer morphism

$$
F H^{*}(W) \stackrel{F j^{!}}{\longrightarrow} F H^{*}(M)
$$

that is associated to a codimension 0 inclusion $W \stackrel{j}{\hookrightarrow} M$, where $M$ has contact type boundary and $W$ has restricted contact type boundary in $W$, with the meaning that the symplectic form admits a primitive defined on the whole of $W$ such that the Liouville vector field is transverse to $\partial W$.

There are two remarks to be made about this transfer morphism. The first remark is that $F j^{!}$is defined only if the manifold $W$ verifies an additional condition on the Floer trajectories ([V2], p. 1000). Following Viterbo, we shall refer to this condition as condition $(A)$. It states that Floer trajectories in $M$ for Hamiltonians of the type below, running between nonconstant 1-periodic orbits contained in $W$, must be entirely contained in $W$. The main point about this condition is that if it is violated then there obviously is a periodic orbit on $\partial W$.

The second remark is that, if $\partial W$ carries no closed characteristic, then the hypothesis of being of restricted contact type in $W$ can be relaxed to contact type, and the morphism $F j$ ! is still defined (condition (A) being assumed).

Here is a brief description of the morphism (4.5) (see also Figure 5). A neighbourhood of $\partial W$ in $W$ is trivialised by the Liouville flow of $W$ as $\partial W \times] 0,1$ ], due to the restricted contact type nature of $\partial W$. Let us denote by $S_{W}$ the coordinate on the second factor. A neighbourhood of $\partial M$ is trivialised in the usual way by the Liouville flow of $M$ as $\partial M \times] 1-\delta, 1], \delta>0$ and we denote by $S$ the coordinate on the second factor. The morphism $F j^{!}$is constructed with the help of a family $H=H_{\mu, \lambda, \epsilon}$ of Hamiltonians that take into account the characteristics on $\partial W$ as well as those on $\partial M$ and which bear the following form: $H=h_{\lambda}\left(S_{W}\right), \epsilon \leq S_{W} \leq 1, h_{\lambda}^{\prime}=\lambda$ and $H=k_{\mu}(S), S \geq 1, k_{\mu}^{\prime}=\mu$, while $H$ is $C^{2}$-close to 0 on $W \backslash \partial W \times[\epsilon, 1]$ and $H=\lambda(1-\epsilon)$ on $M \backslash W$. 
The transfer morphism is induced by the truncation morphism (for a suitable almost complex structure $J$ )

$$
F H_{] 0^{-}, b\right]}^{*}(H, J) \longrightarrow F H_{] a, b]}^{*}(H, J),
$$

with $a=a(\epsilon, \lambda)<0$ negative enough and $b=b(\epsilon, \lambda)>0$ positive enough. An isotopy argument identifies the (inverse) limit following $\epsilon, \lambda$ and $\mu$ in the second term with $F H^{*}(M)$. In the same manner, under the additional hypothesis mentioned above, the first term is identified with $F H_{\left.j 0^{-}, b / \epsilon\right]}^{*}\left(K_{\lambda}\right), K_{\lambda}=k_{\lambda}\left(S_{W}\right)$, $S_{W} \geq 1$ in $\widehat{W}, K_{\lambda}=0$ on $W$. By taking the (inverse) limit following $\lambda$ and $\epsilon$ one gets $F H^{*}(W)$.

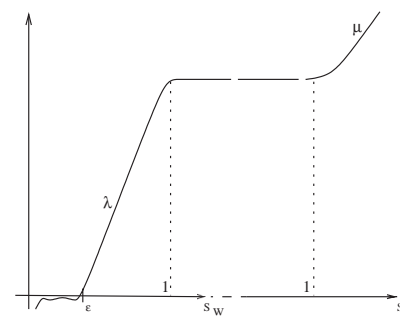

Figure 5: Construction of the transfer morphism [V2]

The fundamental property of the transfer morphism is the commutativity of the following diagram, which is proved by a careful analysis of the various truncation morphisms which are involved [V2] Thm. 3.1. :

$$
\begin{array}{ccc}
F H^{*}(W) & \stackrel{F j^{!}}{\longrightarrow} & F H^{*}(M) \\
c^{*} \downarrow & \downarrow c^{*} \\
H^{n+*}(W, \partial W) & \stackrel{j^{!}}{\longrightarrow} & H^{n+*}(M, \partial M)
\end{array}
$$

This allows one to deduce the following:

Theorem 4.10. ([V2], Thm. 4.1) Let $M$ be a connected symplectic manifold of dimension $2 n$ such that the morphism $F H^{n}(M) \stackrel{c^{*}}{\longrightarrow} H^{2 n}(M, \partial M)$ is not surjective. Any submanifold $W \stackrel{j}{\hookrightarrow} M$ with contact type boundary admits a closed characteristic on $\partial W$.

Proof. Assume that $\partial W$ carries no closed characteristic. Then condition (A) is trivially satisfied and the transfer morphism is defined under the relaxed contact type assumption. The commutative diagram (4.6) therefore holds 
true. The morphism $H^{2 n}(W, \partial W) \longrightarrow H^{2 n}(M, \partial M)$ is bijective if $W$ is connected or surjective if $W$ is disconnected. The hypothesis implies therefore that $F H^{n}(W) \longrightarrow H^{2 n}(W, \partial W)$ is not surjective and we infer the existence of a closed characteristic on $\partial W$. This gives a contradiction and proves that there is always a closed characteristic on $\partial W$.

Remark. If one uses field coefficients and the manifold $M$ is connected, the nonsurjectivity of the morphism $F H^{n}(M) \longrightarrow H^{2 n}(M, \partial M)$ is equivalent to its vanishing.

Let us now mention some interesting cases of manifolds which satisfy case b) of the AWC.

1. We have already seen that

$$
F H^{*}\left(D^{2 n}\right)=0, \quad D^{2 n}=\left\{z \in \mathbb{C}^{n}:|z| \leq 1\right\} .
$$

Through the preceding result, this directly implies Weinstein's conjecture for hypersurfaces of contact type in $\mathbb{C}^{n}$ (the original proof appeared in [V1] ).

2. It is proved in [V2], Thm. 4.2. that a subcritical Stein manifold of dimension $2 n$ (i.e. having the homotopy type of a CW-complex of dimension $n-1$ ) always satisfies case b) of the AWC property: the morphism $F H^{n}(M) \stackrel{c^{*}}{\longrightarrow}$ $H^{2 n}(M, \partial M)$ is not surjective. The same argument as above proves the existence of a closed characteristic on any hypersurface of contact type in a subcritical Stein manifold.

3. The above result can also be deduced as a consequence of a Künneth formula valid in Floer cohomology with field coefficients for a product of manifolds with restricted contact type boundary $[\mathrm{O}], \mathrm{Ch} .2$. There is a commutative diagram where the horizontal arrows are isomorphisms:

$$
\begin{array}{ccc}
\bigoplus_{r+s=k} F H^{r}(M) \otimes F H^{s}(N) & \stackrel{\sim}{\sim} & F H^{k}(M \times N) \\
c^{*} \otimes c^{*} \downarrow & & \downarrow c^{*} \\
\bigoplus_{r+s=k} H^{m+r}(M, \partial M) \otimes H^{n+s}(N, \partial N) & \stackrel{\sim}{\longrightarrow} H^{m+n+k}(M \times N, \partial(M \times N))
\end{array}
$$

Now a theorem of K. Cieliebak [Ci] ensures that a subcritical Stein manifold $M$ is deformation equivalent to a split manifold $\left(N \times \mathbb{C}, \omega_{N} \oplus \omega_{\text {std }}\right)$. The fact that $F H^{*}(\mathbb{C})=0$ allows one to infer the stronger result $F H^{*}(M)=0$.

The commutativity of the diagram (4.7) has not been used in the above argument. Nevertheless it plays a crucial role in the proof of the stability of $\mathrm{AWCb}$ ) under products in the class of restricted contact type symplectic manifolds.

4. I know of only one more case of manifold satisfying case b) of AWC, namely unit disc bundles associated to hermitian line bundles $\mathcal{L}$ with negative 
Chern class $c_{1}(\mathcal{L})=-\lambda[\omega], \lambda>0$ over a symplectic base $(B, \omega)$. These are endowed with a symplectic form that restricts to the area form in the fibers and which equals the pull-back of $\omega$ on the horizontal distribution of a hermitian connection. One can compute in this case $F H^{*}(\mathcal{L})=0$ as a consequence of the existence of a spectral sequence valid in Floer homology [O], Ch. 4.

Let us mention at this point that the only other available explicit computation of Floer homology concerns cotangent bundles ([V3, SW]) :

$$
F H^{*}\left(D T^{*} N\right) \simeq H^{*}(\Lambda N),
$$

where $N$ is a closed Riemannian manifold, $D T^{*} N=\left\{v \in T^{*} N:|v| \leq 1\right\}$ and $\Lambda N$ is the loop space of $N$. The morphism $c^{*}$ coincides, modulo Thom's isomorphism, with the surjection $H^{*}(\Lambda N) \longrightarrow H^{*}(N)$ induced by the inclusion $N \hookrightarrow \Lambda N$. In this situation the argument of Theorem 4.10 does not work anymore in order to prove the Weinstein conjecture in cotangent bundles, but one can nevertheless use the fact that the morphism $F H^{n}\left(D T^{*} N\right) \longrightarrow$ $H^{2 n}\left(D T^{*} N, \partial D T^{*} N\right) \simeq H^{n}(N)$ is surjective in order to prove that there are no exact Lagrangian embeddings $L \hookrightarrow M$ for manifolds $M$ satisfying AWCb) in maximal degree. Here, by exact Lagrangian embedding one means that the Liouville form $\lambda$ on $M$ restricts to an exact form on $L$. Indeed, according to a theorem of Weinstein, an exact Lagrangian embedding would yield an embedding $D T^{*} L \stackrel{j}{\hookrightarrow} M$ with $\partial D T^{*} L$ of restricted contact type in $M$. One can prove that condition (A) is always satisfied in this situation and therefore one would get a commutative diagram

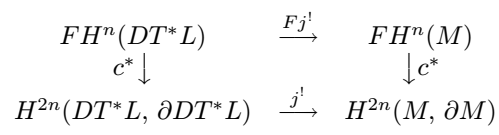

This would imply that $F H^{n}\left(D T^{*} L\right) \longrightarrow H^{2 n}\left(D T^{*} N, \partial D T^{*} N\right)$ is not surjective, a contradiction. We have thus recovered the proof of the following theorem.

Theorem 4.11. [V2] Let $M$ be a manifold with restricted contact type boundary which verifies $A W C b)$ in maximal degree. There is no exact Lagrange embedding $L \hookrightarrow M$.

The above theorem applies in particular for $M$ a subcritical Stein manifold. We also note that the Weinstein conjecture in cotangent bundles is proved in [V2] for simply connected manifolds with the help of an equivariant version of Floer homology.

Before closing this section, let us remark that the preceding results have their analogues in a homological setting. There still is a morphism

$$
H_{n+*}(M, \partial M) \stackrel{c_{*}}{\longrightarrow} F H_{*}(M)
$$


obtained by restricting the range of the action, as well as a transfer morphism

$$
F H_{*}(M) \stackrel{F j_{1}}{\longrightarrow} F H_{*}(W)
$$

which is defined under the same hypothesis as above. This fits into the commutative diagram

$$
\begin{array}{ccc}
F H_{*}(W) & \stackrel{F j_{1}}{\longleftarrow} & F H_{*}(M) \\
c_{*} \uparrow & \uparrow c_{*} \\
H_{n+*}(W, \partial W) \stackrel{j_{!}}{\longleftarrow} & H_{n+*}(M, \partial M)
\end{array}
$$

The proof of these claims is dual to the one in [V2].

\section{Further reading and conclusions}

We have described three constructions and gave an application for each of them. The underlying idea - we have repeated it over and over - is to catch symplectically invariant information about the characteristics in terms of Hamiltonians defined on the whole manifold. A first theme to keep in mind is that there is no "best" construction and that each of the ones we have presented has virtues and shortcomings, which arise from the different behaviours at infinity or near the boundary that one imposes on the admissible Hamiltonians.

Let us mention some material for further reading - here "further" does not mean "harder", but only "different and exploiting similar ideas".

The paper of P. Biran, L. Polterovich and D. Salamon [BPS] develops at least three directions that are closely related to the ideas that we have discussed. The first of them is the construction of Floer homology groups based on the 1-periodic orbits belonging to a given free homotopy class of loops which is not necessarily trivial. The second direction concerns the modification of the class of admissible Hamiltonians. The authors prove existence results for 1periodic orbits of compactly supported Hamiltonians in cotangent bundles of flat tori and in cotangent bundles of negatively curved manifolds, under the only assumption that their value is prescribed and sufficiently large on the zero section. There is an interplay between direct and inverse limits for this class of admissible Hamiltonians and the class of compactly supported ones, which allows the authors to construct relative symplectic capacities for the above types of cotangent bundles. Their computation uses the results of Pozniak [Po] on Floer homology for Hamiltonians whose periodic orbits appear in Morse-Bott nondegenerate families, like the ones in section 3 above.

A generalization of the above results for arbitrary cotangent bundles over closed manifolds is in progress by J. Weber [W], using the computation of truncated Floer homology groups for cotangent bundles [V3, SW]. 
The paper by U. Frauenfelder and F. Schlenk [FrSc] studies the dynamics of compactly supported Hamiltonians on completions of compact manifolds with contact type boundary or products of such objects - split-convex manifolds. The main tool is a kind of symplectic capacity, or selector, associating to any Hamiltonian a value in its spectrum. The selector is defined as the smallest value for which the image of the fundamental class of the manifold through a truncation morphism in Floer homology vanishes. One interesting feature of the authors' construction is that the emphasis is put on the Hamiltonians rather than on some geometric levels, so that they need to consider only slow growths at infinity. Applications include existence of 1-periodic orbits for Hamiltonians having a displaceable support. The main point is that displaceability implies uniform boundedness of the selector for all the iterates of the corresponding Hamiltonian diffeomorphism.

A recent survey of the Weinstein conjecture, including details on the selector method, is provided by the paper of V.L. Ginzburg [Gi].

Let us mention two directions for future research that seem promising. The first concerns the class of admissible Hamiltonians prescribed for each of the homology theories that we have presented. Their behaviour near the boundary $[\mathrm{CFH}, \mathrm{CFHW}]$ or at infinity $[\mathrm{FH} 1, \mathrm{~V} 2]$ is used in a crucial manner in order to obtain a priori bounds on the Floer trajectories but, in some sense, is too rigid. This becomes appearant as soon as one tries to perform geometric constructions on Floer (co)homology, related to additional geometric structure on the underlying manifold. As an example, the difficulty of the proof of the Künneth formula (4.7) lies in the fact that the componentwise sum of two Hamiltonians that are linear at infinity is no longer linear at infinity on the product manifold. It seems to me of interest to enlarge the class of admissible Hamiltonians in the setting of [V2], the main point being to still be able to prove a priori $C^{0}$ bounds. An extension in this sense was accomplished in the dissertation $[\mathrm{O}]$ through Hamiltonians that are asymptotically linear at infinity. This points to the investigation of new instances where the maximum principle can be applied, for example by allowing the almost complex structure to vary as well at infinity.

A second interesting direction of investigation is to clarify the relationship between (truncated) Floer homology and contact homology of the boundary. Contact homology is a recent and powerful invariant that can be defined intrinsically for any contact manifold, in particular for the boundary of contact type of a symplectic manifold. This could lead to obstructions on the topology of symplectic fillings of contact manifolds.

\section{References}

[A] V.I. Arnol'd, Sur une propriété topologique des applications globalement canoniques de la mécanique classique, C.R. Acad. Sc. Paris 261 (1965), 3719-3722.

[BPS] P. Biran, L. Polterovich, D. Salamon, Propagation in Hamiltonian dynamics and relative symplectic homology, Duke Math. J. 119 (2003), no. 1, 65-118. 
[Ci] K. Cieliebak, Subcritical Stein manifolds are split, preprint arXiv:math.DG/0204351.

[CFH] K. Cieliebak, A. Floer, H. Hofer, Symplectic homology II, Math. Z. 218 (1995), 103-122.

[CFHW] K. Cieliebak, A. Floer, H. Hofer, K. Wysocki, Applications of symplectic homology II: Stability of the action spectrum, Math. Z. 223 (1996), 27-45.

[ES] S. Eilenberg, N. Steenrod, Foundations of Algebraic Topology, Princeton Univ. Press, 1952.

[EG] Y. Eliashberg, M. Gromov, Convex symplectic manifolds. In Several complex variables and complex geometry, Part 2 (Santa Cruz, CA, 1989), Proc. Sympos. Pure Math. 52 : 2, AMS, 1991, 135-162.

[F1] A. Floer, The unregularized gradient flow of the symplectic action, Comm. Pure Appl. Math. 41 (1988), 775-813.

[F2] A. Floer, Symplectic fixed points and holomorphic spheres, Comm. Math. Phys. 120 (1989), 575-611.

[F3] A. Floer, Witten's complex and infinite dimensional Morse theory, J. Diff. Geom. 30 (1989), 207-221.

[FH1] A. Floer, H. Hofer, Symplectic homology I, Math. Z. 215 (1994), 37-88.

[FH2] A. Floer, H. Hofer, Coherent orientations for periodic orbit problems in symplectic geometry, Math. Z. 212 (1993), 13-38.

[FHS] A. Floer, H. Hofer, D. Salamon, Transversality in elliptic Morse theory for the symplectic action, Duke Math. J. 80 (1996), 251-292.

[FHW] A. Floer, H. Hofer, K. Wysocki, Applications of symplectic homology I, Math. Z. 217 (1994), 577-606.

[FrSc] U. Frauenfelder, F. Schlenk, Hamiltonian dynamics on convex symplectic manifolds, arXiv:math.SG/0303282, March 2003.

[Gi] V.L. Ginzburg, The Weinstein conjecture and the theorems of nearby and almost existence, arXiv:math.DG/0310330, v2, February 2004.

[He1] D. Hermann, Homologie symplectique et épaisseur de Gromov d'un ouvert de type contact restreint, $\mathrm{PhD}$ thesis, Université Paris-Sud, 1998.

[He2] D. Hermann, Holomorphic curves and Hamiltonian systems in an open set with restricted contact type boundary, Duke Math. J. 103 (2000), 335-374.

[HZ] H. Hofer, E. Zehnder, Symplectic invariants and Hamiltonian dynamics, Birkhäuser, 1994.

[L] F. Laudenbach, On the Thom-Smale complex, appendice à J.M. Bismut, W. Zhang, An extension of a theorem by Cheeger and Müller, Astérisque 205 (1992), 205-214.

[McD] D. McDuff, Symplectic manifolds with contact type boundaries, Invent. Math. 103 (1991), 651-671.

[O] A. Oancea, La suite spectrale de Leray-Serre en homologie de Floer des variétés symplectiques compactes à bord de type contact, $\mathrm{PhD}$ thesis, Université Paris 11 Orsay, September 2003. 
[Po] M. Pozniak, Floer homology, Novikov rings and clean intersections. In Northern California Symplectic Geometry Seminar, AMS Transl. 196, AMS, 1999.

[RS1] J. Robbin, D. Salamon, The Maslov index for paths, Topology 32 (1993), 827844.

[RS2] J. Robbin, D. Salamon, The spectral flow and the Maslov index, Bull. London Math. Soc. 27 (1995), 1-33.

[S1] D. Salamon, Morse theory : the Conley index and Floer homology, Bull. London Math. Soc. 22 (1990), 113-140.

[S2] D. Salamon, Lectures on Floer homology. In Symplectic Geometry and Topology, Y. Eliashberg, L. Traynor, Eds., IAS/Park City Math. Series, vol. 7, AMS, 1999, 143-230.

[SW] D. Salamon, J. Weber, Floer homology and the heat flow, preprint ETH-Zürich (2003), arXiv: math.SG/0304383.

[Sch] M. Schwarz, Morse Homology, Birkhäuser, 1993.

[V1] C. Viterbo, A proof of the Weinstein conjecture in $\mathbb{R}^{2 n}$, Ann. Inst. H. Poincaré, Anal. non-linéaire \& (1987), 337-357.

[V2] C. Viterbo, Functors and computations in Floer homology with applications, I, Geom. Funct. Anal. 9 (1999), 985-1033.

[V3] C. Viterbo, Functors and computations in Floer homology with applications, II, Université Paris-Sud, preprint 98-15 (1998).

[W] J. Weber, Work in progress.

[We] A. Weinstein, On the hypotheses of Rabinowitz' periodic orbit theorems, $J$. Differential Equations 33 (1979), 353-358.

Alexandru OAncea,

Department of Mathematics, ETHZ, 8092 Zürich, Switzerland.

E-mail address: oancea @math.ethz.ch 